\title{
Synthesis of Hydroxamic Acid Derivatives Using Blocked (Masked) O-Isocyanate Precursors
}

Joshua S. Derasp, Erica A. Barbera, Niève R. Séguin, David D. Brzezinski, André M. Beauchemin*

Centre for Catalysis Research and Innovation, Department of Chemistry and Biomolecular Sciences, University of Ottawa, 10 Marie-Curie, Ottawa, ON, K1N 6N5, Canada.

\section{andre.beauchemin@uottawa.ca}

\section{Table of Contents}

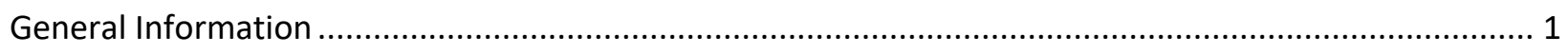

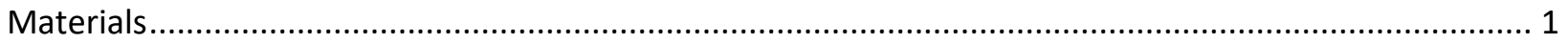

General Procedure

Equation 1: Preparation of 3bt $(1.1 \mathrm{mmol}$ scale) and deprotection .................................................. 9

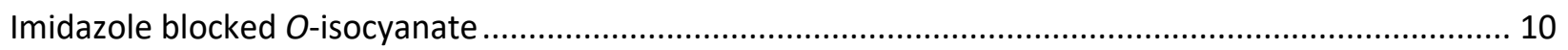

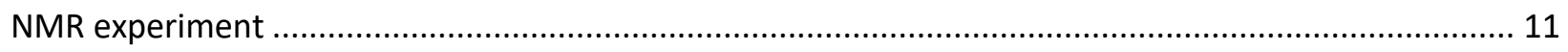

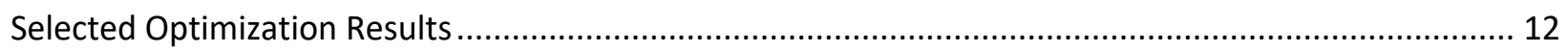

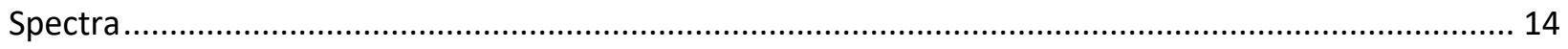

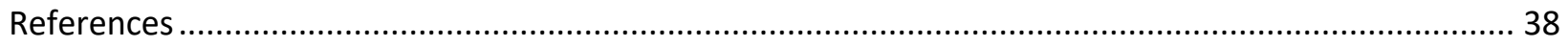

\section{General Information}

Purification of reaction products was carried out by flash column chromatography using Silicycle silica gel $(40-63 \mu \mathrm{m})$, unless otherwise noted. Analytical thin layer chromatography (TLC) was performed on aluminum, cut to size. Visualization was accomplished with UV light followed by staining with a potassium permanganate solution and heating.

${ }^{1} \mathrm{H}$ NMR and ${ }^{13} \mathrm{C}$ NMR spectra were recorded on $300 \mathrm{MHz}, 400 \mathrm{MHz}$, and $500 \mathrm{MHz}$ spectrometers at ambient temperature, unless otherwise indicated. Spectral data was reported in ppm using solvent as the reference $\left(\mathrm{CDCl}_{3}\right.$ at $7.26 \mathrm{ppm}, \mathrm{C}_{6} \mathrm{D}_{6}$ at $7.15 \mathrm{ppm}$ or DMSO- $d_{6}$ at $2.50 \mathrm{ppm}$ for ${ }^{1} \mathrm{H} \mathrm{NMR}$ and $\mathrm{CDCl}_{3}$ at 77.0 ppm or DMSO- $d_{6}$ at 39.43 for ${ }^{13} \mathrm{C}$ NMR). ${ }^{1} \mathrm{H}$ NMR data was reported as: multiplicity (ap = apparent, $b r=$ broad, $\mathrm{s}=$ singlet, $\mathrm{d}=$ doublet, $\mathrm{t}=$ triplet, $\mathrm{q}=$ quartet, quint. = quintet, sext. = sextuplet, sept = septuplet, $\mathrm{m}=$ multiplet), integration and coupling constant(s) in $\mathrm{Hz}$. Infrared (IR) spectra were obtained on neat samples using an Attenuated Total Reflectance Fourier transform infrared spectrometer (ATR-FTIR). Highresolution mass spectroscopy (HRMS) was performed on a mass spectrometer with an electron beam of $70 \mathrm{eV}$ (EI) or Micromass Q-TOF I- Time of Flight Electrospray Ionization mass spectrometer (ESI).

\section{Materials}

Blocked $O$-isocyanates $\mathbf{1 a}, \mathbf{b}, \mathbf{d},{ }^{1}$ c and $\mathbf{e}^{\mathbf{2}}$ were prepared by known methods following literature procedures. Carboxylic acid $\mathbf{2} \mathbf{r}^{3}$ and $\mathbf{2} \mathbf{s}^{4}$ were prepared by known methods following literature procedures. 
Unless otherwise noted, all commercially available materials were purchased from commercial sources and used without further purification. Urea ${ }^{5}$ and isocyanurate ${ }^{6}$ formation was monitored by ${ }^{1} \mathrm{H}$ NMR based on previous literature reports. Suberanilic acid was prepared by known methods following literature procedures. ${ }^{7}$

\section{General Procedure}

General Procedure A: An oven dried microwave vial was charged with a stir bar, carboxylic acid (1.0 equiv.), blocked $O$-isocyanate (3.0 equiv.), $\mathrm{Et}_{3} \mathrm{~N}(0.2$ equiv.) and $\mathrm{MeCN}(0.2 \mathrm{M})$. The vial was sealed under an inert atmosphere of argon with a microwave cap. The vial was immersed in an oil bath at $120^{\circ} \mathrm{C}$ while stirring and monitored by TLC. Upon completion, the crude mixture was concentrated under reduced pressure and purified by silica gel chromatography using an appropriate solvent system to obtain the corresponding hydroxamate.<smiles>O=C(NOCc1ccccc1)c1ccccc1</smiles>

$\mathbf{N}$-(Benzyloxy)benzamide (3aa): Synthesized according to general procedure A using benzoic acid (0.0733 g, $0.600 \mathrm{mmol})$, blocked $O$-isocyanate $1 \mathrm{a}(0.384 \mathrm{~g}, 1.80 \mathrm{mmol}), \mathrm{Et}_{3} \mathrm{~N}(17 \mu \mathrm{L}, 0.20 \mathrm{mmol})$ and $\mathrm{MeCN}(3.0$ $\mathrm{mL}, 0.20 \mathrm{M})$. The vial was sealed with a microwave cap and heated for 5 hours at $120^{\circ} \mathrm{C}$ in an oil bath. The crude mixture was concentrated under reduced pressure and purified by silica gel column chromatography using $40 \%$ EtOAc/Hexanes to generate the pure compound as a white solid $(0.124 \mathrm{~g}, 91$ \%). $\mathrm{TLC} \mathrm{Rf}=0.21$ in 40\% EtOAc/Hexanes. ${ }^{1} \mathrm{H}$ NMR $\left(400 \mathrm{MHz}, \mathrm{DMSO}-d_{6}\right) \delta 11.78(\mathrm{~s}, 1 \mathrm{H}), 7.74(\mathrm{~d}, J=7.0 \mathrm{~Hz}$, $2 \mathrm{H}), 7.58-7.52(\mathrm{~m}, 1 \mathrm{H}), 7.50-7.43(\mathrm{~m}, 4 \mathrm{H}), 7.43-7.33(\mathrm{~m}, 3 \mathrm{H}), 4.93(\mathrm{~s}, 2 \mathrm{H}) .{ }^{13} \mathrm{C} N M R(101 \mathrm{MHz}$, DMSO$\left.d_{6}\right) \delta 164.4(\mathrm{C}), 135.9(\mathrm{C}), 132.3(\mathrm{C}), 131.6(\mathrm{CH}), 128.9(\mathrm{CH}), 128.4(\mathrm{CH}), 128.3(\mathrm{CH}), 128.3(\mathrm{CH}), 127.1(\mathrm{CH})$, $77.0\left(\mathrm{CH}_{2}\right)$. Spectral data was consistent with literature reports. ${ }^{8}$<smiles>CONC(=O)c1ccccc1</smiles>

N-Methoxybenzamide (3ba): Synthesized according to general procedure A using benzoic acid ( $0.0733 \mathrm{~g}$, $0.600 \mathrm{mmol})$, blocked $O$ - isocyanate $1 \mathrm{~b}(0.301 \mathrm{~g}, 1.80 \mathrm{mmol}), \mathrm{Et}_{3} \mathrm{~N}(17 \mu \mathrm{L}, 0.20 \mathrm{mmol})$ and $\mathrm{MeCN}(3.0 \mathrm{~mL}$, $0.20 \mathrm{M})$. The vial was sealed with a microwave cap and heated for 5 hours at $120^{\circ} \mathrm{C}$ in an oil bath. The crude mixture was concentrated under reduced pressure and purified by silica gel column chromatography using $40 \%$ EtOAc/Hexanes to generate the pure compound as a white solid ( $0.0898 \mathrm{~g}$, $99 \%$ ). TLC Rf $=0.54$ in 40\% EtOAc/Hexanes. ${ }^{1} \mathrm{H} \mathrm{NMR} \mathrm{(400} \mathrm{MHz;} \mathrm{CDCl} 3$ ): $\delta 9.91$ (br s, $\left.1 \mathrm{H}\right), 7.75$ (dd, J = 8.32, $1.35 \mathrm{~Hz}, 2 \mathrm{H}), 7.49-7.44(\mathrm{~m}, 1 \mathrm{H}), 7.39-7.34(\mathrm{~m}, 2 \mathrm{H}), 3.80(\mathrm{~s}, 3 \mathrm{H}) .{ }^{13} \mathrm{C} \mathrm{NMR}\left(100 \mathrm{MHz} ; \mathrm{CDCl}_{3}\right) \delta 167.0(\mathrm{C})$, $132.1(\mathrm{C}), 131.9(\mathrm{CH}), 128.7(\mathrm{CH}), 127.3(\mathrm{CH}), 64.4\left(\mathrm{CH}_{3}\right)$. IR (film): 3188, 3180, 2979, 2935, 1638, 1511, $1470,1307,1151 \mathrm{~cm}^{-1}$. Spectral data was consistent with literature reports. ${ }^{9}$ 
<smiles>CCONC(=O)c1ccccc1</smiles>

$\mathrm{N}$-Ethoxybenzamide (3ca): Synthesized according to general procedure A using benzoic acid (0.0733 $\mathrm{g}$, $0.600 \mathrm{mmol})$, blocked $O$-isocyanate $1 \mathrm{c}(0.326 \mathrm{~g}, 1.80 \mathrm{mmol}), \mathrm{Et}_{3} \mathrm{~N}(17 \mu \mathrm{L}, 0.20 \mathrm{mmol})$ and $\mathrm{MeCN}(3.0 \mathrm{~mL}$, $0.20 \mathrm{M}$ ). The vial was sealed with a microwave cap and heated for 24 hours at $120^{\circ} \mathrm{C}$ in an oil bath. The crude mixture was concentrated under reduced pressure and purified by silica gel column chromatography using $40 \%$ EtOAc/Hexanes to generate the pure compound as a white solid $(0.0902 \mathrm{~g}$, $91 \%) . \mathrm{TLC} \mathrm{Rf}=0.28$ in $40 \%$ EtOAc/Hexanes. ${ }^{1} \mathrm{H}$ NMR $\left(400 \mathrm{MHz} ; \mathrm{CDCl}_{3}\right): \delta 9.95(\mathrm{br} \mathrm{s}, 1 \mathrm{H})$, 7.77-7.74 (m, $2 \mathrm{H})$, 7.47-7.42 (m, 1H), 7.37-7.32 (m, 2H), $4.01(\mathrm{q}, 2 \mathrm{H}), 1.23(\mathrm{t}, 3 \mathrm{H}) .{ }^{13} \mathrm{C} \mathrm{NMR}\left(100 \mathrm{MHz} ; \mathrm{CDCl}_{3}\right) \delta 166.6(\mathrm{C})$, $132.1(\mathrm{C}), 131.9(\mathrm{CH}), 128.6(\mathrm{CH}), 127.3(\mathrm{CH}), 72.3\left(\mathrm{CH}_{2}\right), 13.6\left(\mathrm{CH}_{3}\right)$. IR (film): 3193, 2979, 1643, 1577, 1513, 1483, 1383, 1305, $1152 \mathrm{~cm}^{-1}$. HRMS (El-Magn. Sector) m/z: [M]+ Calcd for $\mathrm{C}_{9} \mathrm{H}_{11} \mathrm{NO}_{2} 165.0790$; Found: 165.0790.<smiles>C=CCONC(=O)c1ccccc1</smiles>

$\boldsymbol{N}$-(Allyloxy)benzamide (3da): Synthesized according to general procedure A using benzoic acid $(0.0733 \mathrm{~g}$, $0.600 \mathrm{mmol})$, blocked $O$-isocyanate $1 \mathrm{~d}(0.348 \mathrm{~g}, 1.80 \mathrm{mmol}), \mathrm{Et}_{3} \mathrm{~N}(17 \mu \mathrm{L}, 0.20 \mathrm{mmol})$ and $\mathrm{MeCN}(3.0 \mathrm{~mL}$, $0.20 \mathrm{M})$. The vial was sealed with a microwave cap and heated for 22 hours at $120^{\circ} \mathrm{C}$ in an oil bath. The crude mixture was concentrated under reduced pressure and purified by silica gel column chromatography using $40 \%$ EtOAc/Hexanes to generate the pure compound as an amorphous white solid (0.0755 g, $71 \%)$. TLC Rf $=0.36$ in $40 \%$ EtOAc/Hexanes. ${ }^{1} \mathrm{H}$ NMR $\left(400 \mathrm{MHz} ; \mathrm{CDCl}_{3}\right): \delta 9.51$ (br s, 1H), 7.75$7.72(\mathrm{~m}, 2 \mathrm{H}), 7.50-7.45(\mathrm{~m}, 1 \mathrm{H}), 7.40-7.35(\mathrm{~m}, 2 \mathrm{H}), 6.00$ (ddt, $J=16.9,10.3,6.4 \mathrm{~Hz}, 1 \mathrm{H}), 5.33(\mathrm{dq}, J=17.2$, $1.4 \mathrm{~Hz}, 1 \mathrm{H}), 5.28(\mathrm{ddt}, J=10.3,1.7,0.9 \mathrm{~Hz}, 1 \mathrm{H}), 4.47(\mathrm{~d}, J=6.4 \mathrm{~Hz}, 2 \mathrm{H}) .{ }^{13} \mathrm{C} \mathrm{NMR}\left(100 \mathrm{MHz} ; \mathrm{CDCl}_{3}\right) \delta 166.4$ (C), $132.4(\mathrm{C}), 132.0(\mathrm{CH}), 128.7(\mathrm{CH}), 127.3(\mathrm{CH}), 120.7\left(\mathrm{CH}_{2}\right), 77.5(\mathrm{CH}), 77.3\left(\mathrm{CH}_{2}\right)$. IR (film): 3196, 3061, 3022, 2990, 1643, 1516, 1481, 1307, $1154 \mathrm{~cm}^{-1}$. HRMS (El-Magn. Sector) m/z: [M]+ Calcd for $\mathrm{C}_{10} \mathrm{H}_{11} \mathrm{NO}_{2}$ 177.0790; Found: 177.0805.<smiles>C[Si](C)(C)CCONC(=O)c1ccccc1</smiles>

$\mathrm{N}$-(2-(Trimethylsilyl)ethoxy)benzamide: (3ea): Synthesized according to general procedure A using benzoic acid $(0.0733 \mathrm{~g}, 0.600 \mathrm{mmol})$, blocked $O$-isocyanate $1 \mathrm{e}\left(0.456 \mathrm{~g}, 1.80 \mathrm{mmol}^{2}, \mathrm{Et}_{3} \mathrm{~N}(17 \mu \mathrm{L}, 0.20\right.$ $\mathrm{mmol}$ ) and $\mathrm{MeCN}(3.0 \mathrm{~mL}, 0.20 \mathrm{M})$. The vial was sealed with a microwave cap and heated for 22 hours at $120^{\circ} \mathrm{C}$ in an oil bath. The crude mixture was concentrated under reduced pressure and purified by silica gel column chromatography using $10 \%$ EtOAc/Hexanes to generate the pure compound as a yellow oil (0.114 g, $80 \%$ ). TLC Rf $=0.23$ in 10\% EtOAc/Hexanes. ${ }^{1} \mathrm{H}$ NMR (400 MHz; $\left.\mathrm{CDCl}_{3}\right): \delta 9.44$ (br s, $\left.1 \mathrm{H}\right), 7.77-$ $7.74(\mathrm{~m}, 2 \mathrm{H}), 7.49-7.44(\mathrm{~m}, 1 \mathrm{H}), 7.39-7.35(\mathrm{~m}, 2 \mathrm{H}), 4.04(\mathrm{t}, \mathrm{J}=8.4 \mathrm{~Hz}, 2 \mathrm{H}), 1.04(\mathrm{~m}, 2 \mathrm{H}), 0.00(\mathrm{~s}, 9 \mathrm{H}) .{ }^{13} \mathrm{C}$ NMR (100 MHz; $\left.\mathrm{CDCl}_{3}\right) \delta 166.5(\mathrm{C}), 132.3(\mathrm{C}), 132.0(\mathrm{CH}), 128.7(\mathrm{CH}), 127.3(\mathrm{CH}), 74.6\left(\mathrm{CH}_{2}\right), 16.9\left(\mathrm{CH}_{2}\right)$, $1.3\left(\mathrm{CH}_{3}\right)$. IR (film): 3043, 2953, 2825, 1687, 1593, 1543, 1452, 1369, 1204, $1068 \mathrm{~cm}^{-1}$. HRMS (El-Magn. Sector I) m/z: [M]+ Calcd for $\mathrm{C}_{12} \mathrm{H}_{19} \mathrm{NO}_{2} \mathrm{Si}$ 237.1185; Found: 237.1172 . 
<smiles>CONC(=O)c1ccc(OC)cc1</smiles>

N,4-Dimethoxybenzamide (3bb): Synthesized according to general procedure A using 4-methoxybenzoic acid $(0.0913 \mathrm{~g}, 0.600 \mathrm{mmol})$, blocked $O$-isocyanate $1 \mathrm{~b}(0.301 \mathrm{~g}, 1.80 \mathrm{mmol}), \mathrm{Et}_{3} \mathrm{~N}(17 \mu \mathrm{L}, 0.20 \mathrm{mmol})$ and $\mathrm{MeCN}(3.0 \mathrm{~mL}, 0.20 \mathrm{M})$. The vial was sealed with a microwave cap and heated for 16 hours at $120^{\circ} \mathrm{C}$ in an oil bath. The crude mixture was concentrated under reduced pressure and purified by silica gel column chromatography using 55\% EtOAc/Hexanes to generate the pure compound as a yellow solid (0.0946 g, $87 \%$ ). $\mathrm{TLC} R \mathrm{Rf}=0.27$ in $40 \%$ EtOAc/Hexanes. ${ }^{1} \mathrm{H} \mathrm{NMR}\left(400 \mathrm{MHz} ; \mathrm{CDCl}_{3}\right): \delta 9.56$ (br s, $\left.1 \mathrm{H}\right), 7.74-7.71(\mathrm{~m}, 2 \mathrm{H})$, 6.88-6.84 (m, 2H), $3.81(\mathrm{~s}, 6 \mathrm{H}) .{ }^{13} \mathrm{C}$ NMR (100 MHz; $\left.\mathrm{CDCl}_{3}\right) \delta 166.5$ (C), 162.7 (C), $129.1(\mathrm{CH}), 124.2(\mathrm{C})$, $114.0(\mathrm{CH}), 64.5\left(\mathrm{CH}_{3}\right), 55.5\left(\mathrm{CH}_{3}\right)$. IR (film): 3166, 2927, 1643, 1487, 1255, 1152, 1015 $\mathrm{cm}^{-1}$. Spectral data was consistent with literature reports. ${ }^{10}$<smiles>CONC(=O)c1ccc([N+](=O)[O-])cc1</smiles>

N-Methoxy-4-nitrobenzamide (3bc): Synthesized according to general procedure A using 4-nitrobenzoic acid $(0.100 \mathrm{~g}, 0.600 \mathrm{mmol})$, blocked $O$-isocyanate $1 \mathrm{~b}(0.301 \mathrm{~g}, 1.80 \mathrm{mmol}), \mathrm{Et}_{3} \mathrm{~N}(17 \mu \mathrm{L}, 0.20 \mathrm{mmol})$ and $\mathrm{MeCN}(3.0 \mathrm{~mL}, 0.20 \mathrm{M})$. The vial was sealed with a microwave cap and heated for 24 hours at $120^{\circ} \mathrm{C}$ in an oil bath. The crude mixture was concentrated under reduced pressure and purified by silica gel column chromatography using 50\% EtOAc/Hexanes to generate the pure compound as a white powder $(0.0494 \mathrm{~g}$, $42 \%)$. TLC Rf $=0.26$ in $50 \%$ EtOAc/Hexanes. ${ }^{1} \mathrm{H}$ NMR (400 MHz; DMSO-d 6 ): $\delta 12.07$ (br s, $\left.1 \mathrm{H}\right), 8.33-8.30$

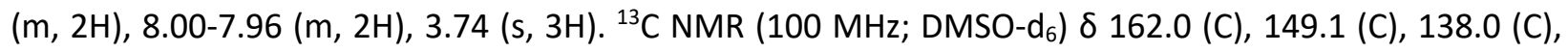
128.5 (CH), 123.6 (CH), 63.3 ( $\mathrm{CH}_{3}$ ). IR (film): 3157, 2988, 2941, 1650, 1598, 1513, 1307, 1295, 1145, 1042 $\mathrm{cm}^{-1}$. Spectral data was consistent with literature reports. ${ }^{8}$<smiles>CONC(=O)c1ccc(NC(C)=O)cc1</smiles>

4-Acetamido- $\boldsymbol{N}$-methoxybenzamide (3bd): Synthesized according to general procedure $A$ using $p$ (acetylamino)benzoic acid $(0.108 \mathrm{~g}, 0.600 \mathrm{mmol})$, blocked $O$-isocyanate $\mathbf{1 b}(0.301 \mathrm{~g}, 1.80 \mathrm{mmol}), \mathrm{Et}_{3} \mathrm{~N}(17$ $\mu \mathrm{L}, 0.20 \mathrm{mmol}$ ) and $\mathrm{MeCN}(3.0 \mathrm{~mL}, 0.20 \mathrm{M})$. The vial was sealed with a microwave cap and heated for 6 hours at $120^{\circ} \mathrm{C}$ in an oil bath. The crude mixture was concentrated under reduced pressure and purified by silica gel column chromatography using $10 \% \mathrm{MeOH} / \mathrm{CH}_{2} \mathrm{Cl}_{2}$ to generate the pure compound as a yellow solid (0.0912 g, $73 \%$ ). TLC Rf $=0.44$ in $10 \% \mathrm{MeOH} / \mathrm{CH}_{2} \mathrm{Cl}_{2} .{ }^{1} \mathrm{H}$ NMR (400 MHz; DMSO-d $)$ ): $\delta 11.60$ (br s, 1H), 10.17 (br s, 1H), 7.71-7.63 (m, 4H), $3.68(\mathrm{~s}, 3 \mathrm{H}), 2.06(\mathrm{~s}, 3 \mathrm{H}) .{ }^{13} \mathrm{C}$ NMR (100 MHz; DMSO-d 6 ) $\delta 168.7$ (C), 142.2 (C), 127.9 (C), $126.4(\mathrm{CH}), 118.2(\mathrm{CH}), 63.2\left(\mathrm{CH}_{3}\right), 24.1\left(\mathrm{CH}_{3}\right)$. IR (film): 3197, 1653, 1596, 1538, 1498, 1297, $1038 \mathrm{~cm}^{-1}$. HRMS (El-Magn. Sector) m/z: [M]+ Calcd for $\mathrm{C}_{10} \mathrm{H}_{12} \mathrm{~N}_{2} \mathrm{O}_{3}$ 208.0848; Found: 208.0836 . 
<smiles>CONC(=O)c1ccc(I)cc1</smiles>

4-lodo- $\mathrm{N}$-methoxybenzamide (3be): Synthesized according to general procedure $\mathrm{A}$ using $\mathrm{p}$-iodobenzoic acid $(0.149 \mathrm{~g}, 0.600 \mathrm{mmol})$, blocked $O$-isocyanate $1 \mathrm{~b}(0.301 \mathrm{~g}, 1.80 \mathrm{mmol}), \mathrm{Et}_{3} \mathrm{~N}(17 \mu \mathrm{L}, 0.20 \mathrm{mmol})$ and MeCN $(3.0 \mathrm{~mL}, 0.20 \mathrm{M})$. The vial was sealed with a microwave cap and heated for 23 hours at $120^{\circ} \mathrm{C}$ in an oil bath. The crude mixture was concentrated under reduced pressure and purified by silica gel column chromatography using $40 \%$ EtOAc/Hexanes to generate the pure compound as a white solid $(0.120 \mathrm{~g}$, $72 \%) . \mathrm{TLC}$ Rf $=0.20$ in 40\% EtOAc/Hexanes. ${ }^{1} \mathrm{H}$ NMR (400 MHz, DMSO- $\left.d_{6}\right) \delta 11.95(\mathrm{~s}, 1 \mathrm{H}), 7.85$ (d, $J=8.3$ $\mathrm{Hz}, 2 \mathrm{H}), 7.55(\mathrm{~d}, J=8.3 \mathrm{~Hz}, 2 \mathrm{H}), 3.69(\mathrm{~s}, 3 \mathrm{H}) .{ }^{13} \mathrm{C}$ NMR (101 MHz, DMSO-d $\left.d_{6}\right) \delta 163.8(\mathrm{C}), 137.8(\mathrm{CH}), 132.1$ (C), $129.5(\mathrm{CH}), 99.8(\mathrm{C}), 63.7\left(\mathrm{CH}_{3}\right)$. HRMS (El-Magn. Sector) m/z: [M]+ Calcd for $\mathrm{C}_{8} \mathrm{H}_{8} \mathrm{NO}_{2} \mathrm{l} 276.9600$; Found: 276.9592.<smiles>CONC(=O)c1ccccc1Cl</smiles>

2-Chloro- $\boldsymbol{N}$-methoxybenzamide (3bf): Synthesized according to general procedure $A$ using $\boldsymbol{O}^{-}$ chlorobenzoic acid $(0.0939 \mathrm{~g}, 0.600 \mathrm{mmol})$, blocked $O$-isocyanate $1 \mathrm{~b}(0.301 \mathrm{~g}, 1.80 \mathrm{mmol}), \mathrm{Et}_{3} \mathrm{~N}(17 \mu \mathrm{L}$, $0.20 \mathrm{mmol})$ and $\mathrm{MeCN}(3.0 \mathrm{~mL}, 0.20 \mathrm{M})$. The vial was sealed with a microwave cap and heated for 23 hours at $120^{\circ} \mathrm{C}$ in an oil bath. The crude mixture was concentrated under reduced pressure and purified by silica gel column chromatography using $40 \%$ EtOAc/Hexanes to generate the pure compound as a white solid (0.0902 g, $81 \%)$. TLC Rf $=0.28$ in $40 \%$ EtOAc/Hexanes. ${ }^{1} \mathrm{H}$ NMR $\left(400 \mathrm{MHz} ; \mathrm{CDCl}_{3}\right): \delta 8.77$ (br s, $1 \mathrm{H}), 7.63(\mathrm{~d}, J=7.5 \mathrm{~Hz}, 1 \mathrm{H}), 7.43-7.39(\mathrm{~m}, 2 \mathrm{H}), 7.38-7.31(\mathrm{~m}, 1 \mathrm{H}), 3.92(\mathrm{~s}, 3 \mathrm{H}) .{ }^{13} \mathrm{C} \mathrm{NMR}\left(100 \mathrm{MHz} ; \mathrm{CDCl}_{3}\right)$ : $\delta 164.5(\mathrm{C}), 132.3(\mathrm{C}), 132.1(\mathrm{C}), 131.3(\mathrm{CH}), 130.6(\mathrm{CH}), 130.4(\mathrm{CH}), 127.3(\mathrm{CH}), 64.9\left(\mathrm{CH}_{3}\right)$. IR (film): 3125, $2932,2808,1635,1530,1432,1310,1031 \mathrm{~cm}^{-1}$. Spectral data was consistent with literature reports. ${ }^{11}$<smiles>CCc1ccccc1C(=O)NOC</smiles>

2-Chloro- $\boldsymbol{N}$-methoxybenzamide (3bg): Synthesized according to general procedure $A$ using 0 ethylbenzoic acid $(0.0901 \mathrm{~g}, 0.600 \mathrm{mmol})$, blocked $O$-isocyanate $1 \mathrm{~b}(0.301 \mathrm{~g}, 1.80 \mathrm{mmol}), \mathrm{Et}_{3} \mathrm{~N}(17 \mu \mathrm{L}, 0.20$ $\mathrm{mmol}$ ) and $\mathrm{MeCN}(3.0 \mathrm{~mL}, 0.20 \mathrm{M})$. The vial was sealed with a microwave cap and heated for 10 hours at $120^{\circ} \mathrm{C}$ in an oil bath. The crude mixture was concentrated under reduced pressure and purified by silica gel column chromatography using $40 \%$ EtOAc/Hexanes providing a white solid as a mixture of the desired product with the oxy-urea by-product $\left(0.0925 \mathrm{~g}, 50 \%{ }^{12}\right)$. TLC Rf $=0.25$ in $40 \%$ EtOAc/Hexanes. ${ }^{1} \mathrm{H}$ NMR $\left(400 \mathrm{MHz} ; \mathrm{CDCl}_{3}\right): \delta 8.95$ (br s, $\left.1 \mathrm{H}\right), 7.35-7.11(\mathrm{~m}, 4 \mathrm{H}), 3.99(\mathrm{~s}, 3 \mathrm{H}), 2.72(\mathrm{q}, J=7.6 \mathrm{~Hz}, 2 \mathrm{H}), 1.18(\mathrm{t}, J=7.5$ $\mathrm{Hz}, 3 \mathrm{H}) .{ }^{13} \mathrm{C} \mathrm{NMR}\left(100 \mathrm{MHz} ; \mathrm{CDCl}_{3}\right) \delta 168.0(\mathrm{C}), 144.1(\mathrm{C}), 132.5(\mathrm{C}), 130.7(\mathrm{CH}), 129.5(\mathrm{CH}), 127.3(\mathrm{CH})$, $125.7(\mathrm{CH}), 65.5\left(\mathrm{CH}_{3}\right), 26.3\left(\mathrm{CH}_{2}\right), 15.8\left(\mathrm{CH}_{3}\right)$. IR (film): 3127, 2929, 2880, 1634, 1524, 1443, 1393, 1314 $\mathrm{cm}^{-1}$. Spectral data was consistent with literature reports. ${ }^{13}$ 


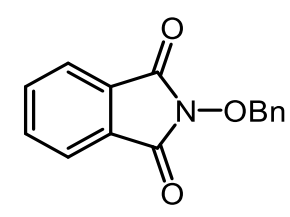

2-(Benzyloxy)isoindoline-1,3-dione (3bi): Synthesized according to general procedure $A$ using phtalic acid $(0.0997 \mathrm{~g}, 0.600 \mathrm{mmol})$, blocked $O$-isocyanate $1 \mathrm{~b}(0.301 \mathrm{~g}, 1.80 \mathrm{mmol}), \mathrm{Et}_{3} \mathrm{~N}(17 \mu \mathrm{L}, 0.20 \mathrm{mmol})$ and $\mathrm{MeCN}$ $(3.0 \mathrm{~mL}, 0.20 \mathrm{M})$. The vial was sealed with a microwave cap and heated for 18 hours at $120^{\circ} \mathrm{C}$ in an oil bath. The crude mixture was concentrated under reduced pressure and purified by silica gel column chromatography using $20 \%$ EtOAc/Hexanes to generate the pure compound as a white powder $(0.0912$ g, $60 \%)$. TLC Rf $=0.47$ in $30 \%$ EtOAc/Hexanes. ${ }^{1} \mathrm{H}$ NMR $\left(400 \mathrm{MHz} ; \mathrm{CDCl}_{3}\right): \delta$ 7.84-7.71 $(\mathrm{m}, 4 \mathrm{H}), 7.55-7.53$ $(\mathrm{m}, 2 \mathrm{H}), 7.41-7.36(\mathrm{~m}, 3 \mathrm{H}), 5.22(\mathrm{~s}, 2 \mathrm{H}) .{ }^{13} \mathrm{C} \mathrm{NMR}\left(100 \mathrm{MHz} ; \mathrm{CDCl}_{3}\right) \delta 163.7(\mathrm{C}), 134.6(\mathrm{C}), 133.9(\mathrm{CH}), 130.1$ (CH), $129.5(\mathrm{C}), 129.1(\mathrm{CH}), 128.7(\mathrm{CH}), 123.7(\mathrm{CH}), 80.1\left(\mathrm{CH}_{2}\right)$. IR (film): 2884 1724, 1460, 1378, 1129, $\mathrm{cm}^{-1}$. HRMS (El-Magn. Sector) m/z: [M]+ Calcd for $\mathrm{C}_{15} \mathrm{H}_{11} \mathrm{NO}_{3}$ 253.0739; Found: 253.0727.<smiles>CONC(=O)c1cccnc1</smiles>

$\mathbf{N}$-Methoxynicotinamide (3bj): Synthesized according to general procedure A using nicotinic acid (0.0739 g, $0.600 \mathrm{mmol})$, blocked $O$-isocyanate $1 \mathbf{b}(0.301 \mathrm{~g}, 1.80 \mathrm{mmol}), \mathrm{Et}_{3} \mathrm{~N}(17 \mu \mathrm{L}, 0.20 \mathrm{mmol})$ and $\mathrm{MeCN}(3.0$ $\mathrm{mL}, 0.20 \mathrm{M}$ ). The vial was sealed with a microwave cap and heated for 23 hours at $120^{\circ} \mathrm{C}$ in an oil bath. The crude mixture was concentrated under reduced pressure and purified by silica gel column chromatography using $60 \%$ EtOAc/Hexanes to generate the pure compound as a green oil $(0.0886 \mathrm{~g}, 97$ \%). TLC Rf $=0.47$ in $10 \% \mathrm{MeOH} / \mathrm{CH}_{2} \mathrm{Cl}_{2} .{ }^{1} \mathrm{H}$ NMR $\left(400 \mathrm{MHz}\right.$, DMSO- $\left.d_{6}\right) \delta 12.06(\mathrm{~s}, 1 \mathrm{H}), 8.92$ (d, $J=3.0 \mathrm{~Hz}$, $1 \mathrm{H}), 8.72(\mathrm{dd}, J=4.8,1.7 \mathrm{~Hz}, 1 \mathrm{H}), 8.12(\mathrm{~d}, J=7.9 \mathrm{~Hz}, 1 \mathrm{H}), 7.51$ (ddd, $J=7.9,4.8,0.9 \mathrm{~Hz}, 1 \mathrm{H}), 3.72(\mathrm{~s}, 1 \mathrm{H})$. ${ }^{13} \mathrm{C}$ NMR (101 MHz, DMSO-d $\left.d_{6}\right) \delta 162.5(\mathrm{C}), 152.3(\mathrm{CH}), 148.1(\mathrm{CH}), 135.0(\mathrm{CH}), 128.0(\mathrm{C}), 123.7(\mathrm{CH}), 63.4$ ( $\left.\mathrm{CH}_{3}\right)$. IR (film): 3161, 2935, 2816, 1648, 1591, 1416, 1304, $1050 \mathrm{~cm}^{-1}$. HRMS (El-Magn. Sector) m/z: [M]+ Calcd for $\mathrm{C}_{7} \mathrm{H}_{8} \mathrm{~N}_{2} \mathrm{O}_{2}$ 152.0586; Found: 152.0569.<smiles>O=C(NCc1ccccc1)c1cnccn1</smiles>

$\mathbf{N}$-(Benzyloxy)pyrazine-2-carboxamide (3ak): Synthesized according to general procedure A using 2pyrazylcarboxylic acid $(0.0745 \mathrm{~g}, 0.600 \mathrm{mmol})$, blocked $O$-isocyanate $1 \mathrm{a}(0.301 \mathrm{~g}, 1.80 \mathrm{mmol}), \mathrm{Et}_{3} \mathrm{~N}(17 \mu \mathrm{L}$, $0.20 \mathrm{mmol})$ and $\mathrm{MeCN}(3.0 \mathrm{~mL}, 0.20 \mathrm{M})$. The vial was sealed with a microwave cap and heated for 2 hours at $120^{\circ} \mathrm{C}$ in an oil bath. The crude mixture was concentrated under reduced pressure and purified by silica gel column chromatography using $20 \% \mathrm{MeOH} / \mathrm{EtOAc}$ to generate the pure compound as a white solid (0.124 g, $90 \%$ ). TLC Rf $=0.35$ in 20\% MeOH/EtOAc. ${ }^{1} \mathrm{H} \mathrm{NMR} \mathrm{(400} \mathrm{MHz;} \mathrm{CDCl} 3$ ): $\delta 10.25$ (br s, $\left.1 \mathrm{H}\right), 9.33$ (s, $1 \mathrm{H}), 8.68(\mathrm{~s}, 1 \mathrm{H}), 8.39(\mathrm{~s}, 1 \mathrm{H}), 7.42-7.39(\mathrm{~m}, 2 \mathrm{H}), 7.35-7.30(\mathrm{~m}, 3 \mathrm{H}), 5.03(\mathrm{~s}, 2 \mathrm{H}) .{ }^{13} \mathrm{C} \mathrm{NMR}\left(100 \mathrm{MHz} ; \mathrm{CDCl}_{3}\right)$ $\delta 160.4(\mathrm{C}), 147.8(\mathrm{C}), 144.2(\mathrm{CH}), 143.8(\mathrm{CH}), 142.7(\mathrm{CH}), 135.0(\mathrm{C}), 129.3(\mathrm{CH}), 128.9(\mathrm{CH}), 128.7(\mathrm{CH})$, $78.7\left(\mathrm{CH}_{2}\right)$. IR (film): 3154, 2923, 1686, 1482, 1396, 1286, $1016 \mathrm{~cm}^{-1}$. HRMS (El-Magn. Sector) m/z: [M]+ Calcd for $\mathrm{C}_{! 2} \mathrm{H}_{11} \mathrm{~N}_{3} \mathrm{O}_{2}$ 229.0851; Found: 229.0632 . 
<smiles>CONC(=O)c1ccsc1</smiles>

$\mathbf{N}$-Methoxythiophene-3-carboxamide (3bl): Synthesized according to general procedure A using 3thiophene carboxylic acid $(0.0769 \mathrm{~g}, 0.600 \mathrm{mmol})$, blocked $O$-isocyanate $1 \mathbf{b}(0.301 \mathrm{~g}, 1.80 \mathrm{mmol}), \mathrm{Et}_{3} \mathrm{~N}(17$ $\mu \mathrm{L}, 0.20 \mathrm{mmol})$ and $\mathrm{MeCN}(3.0 \mathrm{~mL}, 0.20 \mathrm{M})$. The vial was sealed with a microwave cap and heated for 15 hours at $120^{\circ} \mathrm{C}$ in an oil bath. The crude mixture was concentrated under reduced pressure and purified by silica gel column chromatography using $5 \% \mathrm{EtOAc} / \mathrm{CH}_{2} \mathrm{Cl}_{2}$ to generate the pure compound as clear crystals (0.0905 g, $96 \%)$. TLC Rf $=0.25$ in $5 \% \mathrm{EtOAc} / \mathrm{CH}_{2} \mathrm{Cl}_{2} .{ }^{1} \mathrm{H}$ NMR $\left(400 \mathrm{MHz}, \mathrm{DMSO}-d_{6}\right) \delta 11.61(\mathrm{~s}, 1 \mathrm{H})$, $8.08(\mathrm{dd}, J=3.0,1.3 \mathrm{~Hz}, 1 \mathrm{H}), 7.62(\mathrm{dd}, J=5.0,2.9 \mathrm{~Hz}, 1 \mathrm{H}), 7.41(\mathrm{dd}, J=5.1,1.3 \mathrm{~Hz}, 1 \mathrm{H}), 3.69(\mathrm{~s}, 3 \mathrm{H}) .{ }^{13} \mathrm{C}$ NMR (101 MHz, DMSO- $\left.d_{6}\right) \delta 160.0(\mathrm{C}), 134.7(\mathrm{C}), 129.1(\mathrm{CH}), 127.1(\mathrm{CH}), 126.2(\mathrm{CH}), 63.4\left(\mathrm{CH}_{3}\right)$. IR (film): 3231, 3087, 2923, 1642, 1524, 1419, 1289, $1026 \mathrm{~cm}^{-1}$. HRMS (El-Magn. Sector) m/z: [M]+ Calcd for $\mathrm{C}_{6} \mathrm{H}_{7} \mathrm{NO}_{2} \mathrm{~S}$ 157.0198; Found: 157.0205.

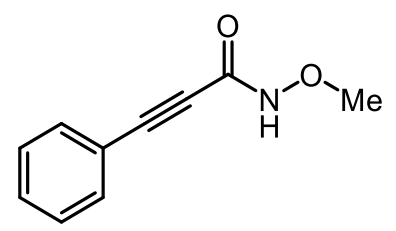

N-Methoxy-3-phenylpropiolamide (3bm): Synthesized according to general procedure A using phenylpropiolic acid $(0.0877 \mathrm{~g}, 0.600 \mathrm{mmol})$, blocked $O$-isocyanate $\mathbf{1 b}(0.301 \mathrm{~g}, 1.80 \mathrm{mmol})$, triethylamine $(17 \mu \mathrm{L}, 0.20 \mathrm{mmol})$ and $\mathrm{MeCN}(3.0 \mathrm{~mL}, 0.20 \mathrm{M})$. The vial was sealed with a microwave cap and heated for 24 hours at $120^{\circ} \mathrm{C}$ in an oil bath. The crude mixture was concentrated under reduced pressure and purified by silica gel column chromatography using $30 \%$ EtOAc/Hexanes to generate the pure compound as a yellow oil (0.0547 g, $52 \%)$. TLC Rf $=0.22$ in 30\% EtOAc/Hexanes. ${ }^{1} \mathrm{H}$ NMR (400 MHz; DMSO-d 6 ): $\delta 11.04$ (br s, 1H), 7.43-7.38 (m, 2H), 7.26-7.22 (m, 1H), 7.16-7.12 (m, 2H), 3.65 (s, 3H). ${ }^{13} \mathrm{C} \mathrm{NMR} \mathrm{(100} \mathrm{MHz;} \mathrm{DMSO-}$ $\left.\mathrm{d}_{6}\right) \delta 163.7$ (C), 134.6 (C), 133.9 (C), 130.1 (C), 129.5 (CH), 128.7 (CH), $123.7(\mathrm{CH}), 80.1\left(\mathrm{CH}_{3}\right)$. IR (film): 3407, 2922, 2852, 2116, 1741, 1458, 1376, $1062 \mathrm{~cm}^{-1}$. HRMS (El-Magn. Sector) m/z: [M]+ Calcd for $\mathrm{C}_{10} \mathrm{H}_{9} \mathrm{NO}_{2}$ 175.0633; Found: 175.0667.<smiles>CONC(=O)/C=C/c1ccccc1</smiles>

$\mathbf{N}$-Methoxycinnamamide (3bn): Synthesized according to general procedure A using (E)-3-phenylacrylic acid $(0.0889 \mathrm{~g}, 0.600 \mathrm{mmol})$, blocked $O$-isocyanate $1 \mathrm{~b}(0.301 \mathrm{~g}, 1.80 \mathrm{mmol}), \mathrm{Et}_{3} \mathrm{~N}(17 \mu \mathrm{L}, 0.20 \mathrm{mmol})$ and $\mathrm{MeCN}(3.0 \mathrm{~mL}, 0.20 \mathrm{M})$. The vial was sealed with a microwave cap and heated for 16 hours at $120^{\circ} \mathrm{C}$ in an oil bath. The crude mixture was concentrated under reduced pressure and purified by silica gel column chromatography using $25 \%$ EtOAc/Hexanes to generate the pure compound as a white solid (0.0893 g, $84 \%$ ). TLC Rf $=0.30$ in 40\% EtOAc/Hexanes. ${ }^{1} \mathrm{H}$ NMR (400 MHz; DMSO-d 6 ): $\delta 11.30$ (br s, 1H), $7.58(\mathrm{~d}, J=$ $7.1 \mathrm{~Hz}, 2 \mathrm{H}), 7.51(\mathrm{~d}, J=15.8 \mathrm{~Hz}, 1 \mathrm{H}), 7.44-7.36(\mathrm{~m}, 3 \mathrm{H}), 6.42(\mathrm{~d}, J=15.9 \mathrm{~Hz}, 1 \mathrm{H}), 3.67(\mathrm{~s}, 3 \mathrm{H}) .{ }^{13} \mathrm{C} \mathrm{NMR}(100$ $\left.\mathrm{MHz} ; \mathrm{CDCl}_{3}\right) \delta 162.7(\mathrm{C}), 139.6(\mathrm{C}), 134.6(\mathrm{CH}), 129.7(\mathrm{CH}), 128.9(\mathrm{CH}), 127.6(\mathrm{CH}), 118.5(\mathrm{CH}), 63.4\left(\mathrm{CH}_{3}\right) . \mathrm{IR}$ (film): 3130, 2981, 2941, 1615, 1519, 1448, 1339, 1217, $1057 \mathrm{~cm}^{-1}$. HRMS (El-Magn. Sector) m/z: [M]+ Calcd for $\mathrm{C}_{10} \mathrm{H}_{11} \mathrm{NO}_{2}$ 177.0790; Found: 177.0782 . 
<smiles>CONC(=O)CCc1ccccc1</smiles>

N-Methoxy-3-phenylpropanamide (3bo): Synthesized according to general procedure $A$ using 3phenylpropionic acid $(0.0901 \mathrm{~g}, 0.600 \mathrm{mmol})$, blocked $O$-isocyanate $1 \mathrm{~b}(0.301 \mathrm{~g}, 1.80 \mathrm{mmol}), \mathrm{Et}_{3} \mathrm{~N}(17 \mu \mathrm{L}$, $0.20 \mathrm{mmol}$ ) and $\mathrm{MeCN}(3.0 \mathrm{~mL}, 0.20 \mathrm{M})$. The vial was sealed with a microwave cap and heated for 16 hours at $120^{\circ} \mathrm{C}$ in an oil bath. The crude mixture was concentrated under reduced pressure and purified by silica gel column chromatography using $40 \%$ EtOAc/Hexanes to generate the pure compound as a yellow oil (0.0936 g, $87 \%)$. TLC Rf $=0.24$ in $40 \%$ EtOAc/Hexanes. $\left.{ }^{1} \mathrm{H} \mathrm{NMR} \mathrm{(400} \mathrm{MHz;} \mathrm{CDCl}_{3}\right): \delta 10.10$ (br s, $1 \mathrm{H}), 7.26-7.22(\mathrm{~m}, 2 \mathrm{H}), 7.19-7.15(\mathrm{~m}, 3 \mathrm{H}), 3.62(\mathrm{~s}, 3 \mathrm{H}), 2.94(\mathrm{t}, J=8.7 \mathrm{~Hz}, 2 \mathrm{H}), 2.41(\mathrm{t}, J=7.8 \mathrm{~Hz}, 2 \mathrm{H}) .{ }^{13} \mathrm{C}$ NMR (100 MHz; CDCl $) \delta 170.3(\mathrm{C}), 140.5(\mathrm{C}), 128.5(\mathrm{CH}), 128.4(\mathrm{CH}), 126.3(\mathrm{CH}), 64.0\left(\mathrm{CH}_{3}\right), 34.8\left(\mathrm{CH}_{2}\right)$, $31.5\left(\mathrm{CH}_{2}\right)$. IR (film): 3173, 2969, 1649, 1490, 1438, 1149, $1065 \mathrm{~cm}^{-1}$. Spectral data was consistent with literature reports. ${ }^{14}$<smiles>CONC(=O)C(C)(C)CCCOc1cc(C)ccc1C</smiles>

5-(2,5-Dimethylphenoxy)- $\mathrm{N}$-methoxy-2,2-dimethylpentanamide (3bp): Synthesized according to general procedure A using gemfibrozil $(0.150 \mathrm{~g}, 0.600 \mathrm{mmol})$, blocked $O$-isocyanate $\mathbf{1 b}(0.301 \mathrm{~g}, 1.80 \mathrm{mmol}), \mathrm{Et}_{3} \mathrm{~N}$ $(17 \mu \mathrm{L}, 0.20 \mathrm{mmol})$ and $\mathrm{MeCN}(3.0 \mathrm{~mL}, 0.20 \mathrm{M})$. The vial was sealed with a microwave cap and heated for 23 hours at $120^{\circ} \mathrm{C}$ in an oil bath. The crude mixture was concentrated under reduced pressure and purified by silica gel column chromatography using $20 \%$ EtOAc/Hexanes to generate the pure compound as a yellow oil $(0.166 \mathrm{~g}, 99 \%)$. TLC Rf $=0.28$ in $20 \%$ EtOAc/Hexanes. ${ }^{1} \mathrm{H}$ NMR $(400 \mathrm{MHz}$; DMSO-d 6 ): $\delta 10.89(\mathrm{br}$ $\mathrm{s}, 1 \mathrm{H}), 6.97(\mathrm{~d}, J=7.5 \mathrm{~Hz}, 1 \mathrm{H}), 6.69(\mathrm{~s}, 1 \mathrm{H}), 6.61(\mathrm{~d}, J=7.3 \mathrm{~Hz}, 1 \mathrm{H}), 3.88(\mathrm{t}, J=5.8 \mathrm{~Hz}, 2 \mathrm{H}), 3.55(\mathrm{~s}, 3 \mathrm{H}), 2.24$ $(\mathrm{s}, 3 \mathrm{H}), 2.08(\mathrm{~s}, 3 \mathrm{H}), 1.58(\mathrm{~m}, 4 \mathrm{H}), 1.08(\mathrm{~s}, 6 \mathrm{H}) .{ }^{13} \mathrm{C} \mathrm{NMR}(100 \mathrm{MHz}$; DMSO-d 6 ) $\delta 173.5(\mathrm{C}), 156.4(\mathrm{C}), 136.0$ (C), $130.0(\mathrm{C}), 122.5(\mathrm{CH}), 120.5(\mathrm{CH}), 112.0(\mathrm{CH}), 67.6\left(\mathrm{CH}_{3}\right), 62.7\left(\mathrm{CH}_{3}\right), 40.2\left(\mathrm{CH}_{2}\right), 36.8(\mathrm{C}), 24.8\left(\mathrm{CH}_{2}\right)$, $24.5\left(\mathrm{CH}_{2}\right), 21.0\left(\mathrm{CH}_{3}\right), 15.5\left(\mathrm{CH}_{3}\right)$. IR (film): 3215, 2924, 2867, 2815, 1646, 1507, 1262, 1128, $1039 \mathrm{~cm}^{-1}$. HRMS (El-Magn. Sector) m/z: [M]+ Calcd for $\mathrm{C}_{16} \mathrm{H}_{25} \mathrm{NO}_{3}$ 279.1834; Found: 279.1827.<smiles>CONC(=O)CC(C)CCC=C(C)C</smiles>

N-Methoxy-3,7-dimethyloct-6-enamide (3bq): Synthesized according to general procedure A using citronellic acid $(0.102 \mathrm{~g}, 0.600 \mathrm{mmol})$, blocked $O$-isocyanate $1 \mathrm{~b}\left(0.301 \mathrm{~g}, 1.80 \mathrm{mmol}^{2}\right), \mathrm{Et}_{3} \mathrm{~N}(17 \mu \mathrm{L}, 0.20$ $\mathrm{mmol})$ and $\mathrm{MeCN}(3.0 \mathrm{~mL}, 0.20 \mathrm{M})$. The vial was sealed with a microwave cap and heated for 5 hours at $120^{\circ} \mathrm{C}$ in an oil bath. The crude mixture was concentrated under reduced pressure and purified by silica gel column chromatography using $20 \% \mathrm{EtOAc} / \mathrm{CH}_{2} \mathrm{Cl}_{2}$ to generate the pure compound as a colourless oil (0.104 g, 87\%). $\mathrm{TLC} \mathrm{Rf}=0.20$ in 20\% EtOAc/ $\mathrm{CH}_{2} \mathrm{Cl}_{2}{ }^{1} \mathrm{H} \mathrm{NMR}\left(400 \mathrm{MHz} ; \mathrm{CDCl}_{3}\right): \delta 10.11$ (br s, $\left.1 \mathrm{H}\right), 5.01$ (t, J $=6.9 \mathrm{~Hz}, 1 \mathrm{H}), 3.69(\mathrm{~s}, 3 \mathrm{H}), 2.09(\mathrm{dd}, J=13.3,5.5 \mathrm{~Hz}, 1 \mathrm{H}), 1.92(\mathrm{tdd}, J=21.2,9.8,5.5 \mathrm{~Hz}, 4 \mathrm{H}), 1.61(\mathrm{~s}, 3 \mathrm{H})$, $1.53(\mathrm{~s}, 3 \mathrm{H}), 1.37-1.28(\mathrm{~m}, 1 \mathrm{H}), 1.21-1.11(\mathrm{~m}, 1 \mathrm{H}), 0.89(\mathrm{~d}, J=6.5 \mathrm{~Hz}, 3 \mathrm{H}) .{ }^{13} \mathrm{C} \mathrm{NMR}\left(100 \mathrm{MHz} ; \mathrm{CDCl}_{3}\right) \delta$ $170.6(\mathrm{C}), 131.5(\mathrm{C}), 124.3(\mathrm{CH}), 64.0\left(\mathrm{CH}_{3}\right), 40.5\left(\mathrm{CH}_{3}\right), 36.9\left(\mathrm{CH}_{3}\right), 30.3\left(\mathrm{CH}_{3}\right), 25.7\left(\mathrm{CH}_{2}\right), 25.5(\mathrm{CH}), 19.3$ $\left(\mathrm{CH}_{2}\right), 17.7\left(\mathrm{CH}_{2}\right)$. IR (film): 3172, 2962, 2916, 1651, 1510, 1439, 1377, $1052 \mathrm{~cm}^{-1}$. HRMS (ESI-TOF) m/z: [M+Na]+ Calcd for $\mathrm{C}_{11} \mathrm{H}_{21} \mathrm{NO}_{2} \mathrm{Na}$ 222.1470; Found: 222.1469 . 
<smiles>CONC(=O)CCCCCCC(=O)Nc1ccccc1</smiles>

$\boldsymbol{N}^{\mathbf{1}}$-Methoxy- $\boldsymbol{N}^{\mathbf{8}}$-phenyloctanediamide (3br): Synthesized according to general procedure A using 8-oxo8-(phenylamino)octanoic acid $(0.0733 \mathrm{~g}, 0.600 \mathrm{mmol})$, blocked $O$-isocyanate $1 \mathrm{~b}\left(0.301 \mathrm{~g}, 1.80 \mathrm{mmol}^{\text {) }}, \mathrm{Et}_{3} \mathrm{~N}\right.$ $(17 \mu \mathrm{L}, 0.20 \mathrm{mmol})$ and $\mathrm{MeCN}(3.0 \mathrm{~mL}, 0.20 \mathrm{M})$. The vial was sealed with a microwave cap and heated for 2 hours at $120^{\circ} \mathrm{C}$ in an oil bath. The crude mixture was concentrated under reduced pressure and purified by silica gel column chromatography using $100 \%$ EtOAc/Hexanes to generate the pure compound as a white solid (0.124 g, $74 \%)$. TLC Rf $=0.25$ in $100 \%$ EtOAc/Hexanes. ${ }^{1} \mathrm{H}$ NMR (400 MHz; DMSO-d 6 ): $\delta 810.93$ (br s, 1H), $9.83(\mathrm{br} \mathrm{s}, 1 \mathrm{H}), 7.60-7.57(\mathrm{~m}, 2 \mathrm{H}), 7.29-7.25(\mathrm{~m}, 2 \mathrm{H}), 7.03-6.99(\mathrm{~m}, 1 \mathrm{H}), 3.56(\mathrm{~s}, 3 \mathrm{H}), 2.29(\mathrm{t}, J=$ $7.4 \mathrm{~Hz}, 2 \mathrm{H}), 1.93(\mathrm{t}, \mathrm{J}=7.4 \mathrm{~Hz}, 2 \mathrm{H}), 1.61-1.46(\mathrm{~m}, 4 \mathrm{H}), 1.28-1.27(\mathrm{~m}, 4 \mathrm{H}) .{ }^{13} \mathrm{C} \mathrm{NMR}(400 \mathrm{MHz}$; DMSO-d $) \delta$ $171.1(\mathrm{C}), 169.0(\mathrm{C}), 139.3(\mathrm{C}), 128.6(\mathrm{CH}), 122.8(\mathrm{CH}), 119.0(\mathrm{CH}), 63.0\left(\mathrm{CH}_{3}\right), 36.3\left(\mathrm{CH}_{2}\right), 32.2\left(\mathrm{CH}_{2}\right), 28.4$ $\left(\mathrm{CH}_{2}\right), 28.3\left(\mathrm{CH}_{2}\right), 24.9\left(\mathrm{CH}_{2}\right), 24.8\left(\mathrm{CH}_{2}\right)$. IR (film): 3297, 3253, 3197, 2923, 2849, 1695, 1655, 1597, 1540, 1439, 1382, 1247, 1190, $1060 \mathrm{~cm}^{-1}$. HRMS (ESI-TOF) m/z: [M+Na]+ Calcd for $\mathrm{C}_{15} \mathrm{H}_{22} \mathrm{~N}_{2} \mathrm{O}_{3} \mathrm{Na} 301.1528$; Found: 301.1521.

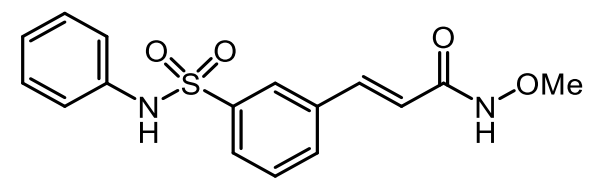

(E)-N-Methoxy-3-(3-(N-phenylsulfamoyl)phenyl)acrylamide (3bs): Synthesized according to general procedure A using (E)-3-(3-( $N$-phenylsulfamoyl)phenyl)acrylic acid $(0.182 \mathrm{~g}, 0.600 \mathrm{mmol})$, blocked $O$ isocyanate $1 \mathbf{b}(0.301 \mathrm{~g}, 1.800 \mathrm{mmol}), \mathrm{Et}_{3} \mathrm{~N}(17 \mu \mathrm{L}, 0.20 \mathrm{mmol})$ and $\mathrm{MeCN}(3.0 \mathrm{~mL}, 0.20 \mathrm{M})$. The vial was sealed with a microwave cap and heated for 19 hours at $120^{\circ} \mathrm{C}$ in an oil bath. The crude mixture was concentrated under reduced pressure and purified by silica gel column chromatography using $100 \%$ EtOAc to generate the pure compound as a white solid $(0.158 \mathrm{~g}, 79 \%)$. TLC Rf $=0.35$ in $100 \%$ EtOAc. ${ }^{1} \mathrm{H}$ NMR (400 MHz; DMSO-d 6 ): $\delta 811.36$ (br s, 1H), 10.35 (br s, 1H) $7.93(\mathrm{~s}, 1 \mathrm{H}), 7.80$ (d, J = 7.7 Hz, 1H), 7.72 $(\mathrm{d}, J=7.7 \mathrm{~Hz}, 1 \mathrm{H}), 7.57(\mathrm{t}, J=7.8 \mathrm{~Hz}, 1 \mathrm{H}), 7.51(\mathrm{~d}, J=15.9 \mathrm{~Hz}, 1 \mathrm{H}), 7.25-7.20(\mathrm{~m}, 2 \mathrm{H}), 7.10(\mathrm{dd}, J=8.6,1.3$ $\mathrm{Hz}, 2 \mathrm{H}), 7.05-7.00(\mathrm{~m}, 1 \mathrm{H}), 6.46(\mathrm{~d}, J=15.9 \mathrm{~Hz}, 1 \mathrm{H}), 3.67(\mathrm{~s}, 3 \mathrm{H}) .{ }^{13} \mathrm{C}$ NMR $(400 \mathrm{MHz}$; DMSO-d 6 ) $\delta 162.0(\mathrm{C})$, $140.3(\mathrm{C}), 137.7(\mathrm{C}), 137.5(\mathrm{C}), 135.6(\mathrm{CH}), 132.0(\mathrm{CH}), 130.0(\mathrm{CH}), 129.2(\mathrm{CH}), 127.4(\mathrm{CH}), 125.1(\mathrm{CH}), 124.4$ (CH), $120.8(\mathrm{CH}), 120.4(\mathrm{CH}), 63.4\left(\mathrm{CH}_{3}\right)$. IR (film): 3557, 3074, 2890, 1664, 1627, 1493, 1338, 1306, 1150, $1063 \mathrm{~cm}^{-1}$. HRMS (El-Magn. Sector) m/z: [M]+ Calcd for $\mathrm{C}_{16} \mathrm{H}_{16} \mathrm{~N}_{2} \mathrm{O}_{4} \mathrm{~S} 332.0831$; Found: 332.0839 .

\section{Equation 1: Preparation of 3bt (1.1 mmol scale) and deprotection}<smiles>O=C(CCCCCCC(=O)Nc1ccccc1)NOCc1ccccc1</smiles>

$\boldsymbol{N}^{1}$-(Benzyloxy)- $\boldsymbol{N}^{8}$-phenyloctanediamide (3bt): Synthesized according to general procedure A using 8oxo-8-(phenylamino)octanoic acid $(0.265 \mathrm{~g}, 1.10 \mathrm{mmol})$, blocked $O$-isocyanate $1 \mathrm{a}(0.803 \mathrm{~g}, 3.30 \mathrm{mmol})$, $\mathrm{Et}_{3} \mathrm{~N}(30.6 \mu \mathrm{L}, 0.220 \mathrm{mmol})$ and $\mathrm{MeCN}(5.5 \mathrm{~mL})$. The vial was sealed with a microwave cap and heated for 18 hours at $120^{\circ} \mathrm{C}$ in an oil bath. The crude mixture was concentrated under reduced pressure and purified by a recrystallization with EtOAc to yield a white solid $\left(0.750 \mathrm{~g}\right.$, 71\%). TLC Rf $=0.45$ in $5 \% \mathrm{MeOH} / \mathrm{CH}_{2} \mathrm{Cl}_{2}$. ${ }^{1} \mathrm{H}$ NMR $\left(500 \mathrm{MHz}, \mathrm{DMSO}-d_{6}\right) \delta 10.94(\mathrm{~s}, 1 \mathrm{H}), 9.84(\mathrm{~s}, 1 \mathrm{H}), 7.59(\mathrm{~d}, J=8.2 \mathrm{~Hz}, 2 \mathrm{H}), 7.42-7.31(\mathrm{~m}, 5 \mathrm{H}), 7.32$ $-7.24(\mathrm{~m}, 2 \mathrm{H}), 7.02(\mathrm{tt}, J=7.4,1.3 \mathrm{~Hz}, 1 \mathrm{H}), 4.78(\mathrm{~s}, 2 \mathrm{H}), 2.29(\mathrm{t}, J=7.4 \mathrm{~Hz}, 2 \mathrm{H}), 1.95(\mathrm{t}, J=7.3 \mathrm{~Hz}, 2 \mathrm{H}), 1.57$ $(p, J=7.4 \mathrm{~Hz}, 2 \mathrm{H}), 1.50(\mathrm{p}, J=7.4 \mathrm{~Hz}, 2 \mathrm{H}), 1.34-1.15(\mathrm{~m}, J=4.3,3.8 \mathrm{~Hz}, 4 \mathrm{H}) .{ }^{13} \mathrm{C}$ NMR $(126 \mathrm{MHz}$, DMSO- 
$\left.d_{6}\right) \delta 171.2(\mathrm{C}), 169.4(\mathrm{C}), 139.4(\mathrm{C}), 136.1(\mathrm{C}) 128.8(\mathrm{CH}), 128.6(\mathrm{CH}), 128.3(\mathrm{CH}), 128.2(\mathrm{CH}), 122.9(\mathrm{CH})$, $119.0(\mathrm{CH}), 76.7\left(\mathrm{CH}_{2}\right), 36.4\left(\mathrm{CH}_{2}\right), 32.2\left(\mathrm{CH}_{2}\right), 28.4\left(\mathrm{CH}_{2}\right), 28.3\left(\mathrm{CH}_{2}\right), 25.0\left(\mathrm{CH}_{2}\right), 24.9\left(\mathrm{CH}_{2}\right)$. Spectral data obtained was consistent with literature data. ${ }^{15}$<smiles>O=C(CCCCCCC(=O)Nc1ccccc1)NO</smiles>

$\boldsymbol{N}^{1}$-Hydroxy- $\boldsymbol{N}^{8}$-phenyloctanediamide (4): Synthesized using $\boldsymbol{N}^{1}$-(benzyloxy)- $\boldsymbol{N}^{8}$-phenyloctanediamide $(0.212 \mathrm{~g}, 0.600 \mathrm{mmol})$, methanol $(6.0 \mathrm{~mL}), \mathrm{Pd} / \mathrm{C}(0.120 \mathrm{~g}, 20 \%)$. The reaction vessel was sealed then purged under argon followed by vacuum $3 x$. A balloon with $\mathrm{H}_{2}$ gas was then used and a vacuum/purge procedure was then done $3 \mathrm{x}$. The reaction was then left to stir for 4 hours until completion by TLC. After completion, the reaction was then filtered through a pad of Celite. The reaction was then dried down to yield the pure compound as an off-white solid (0.155 g, 98\%). TLC $\mathrm{R}_{\mathrm{F}}=0.14$ in $10 \% \mathrm{MeOH} / \mathrm{CH}_{2} \mathrm{Cl}_{2} .{ }^{1} \mathrm{H} \mathrm{NMR}(300 \mathrm{MHz}$, DMSO- $\left.d_{6}\right) \delta 10.32(\mathrm{~s}, 1 \mathrm{H}), 9.84(\mathrm{~s}, 1 \mathrm{H}), 7.58(\mathrm{~d}, J=8.0 \mathrm{~Hz}, 2 \mathrm{H}), 7.27(\mathrm{t}, J=7.8 \mathrm{~Hz}, 2 \mathrm{H}), 7.01(\mathrm{t}, J=7.3 \mathrm{~Hz}$, $1 \mathrm{H}), 2.27(\mathrm{t}, J=7.4 \mathrm{~Hz}, 2 \mathrm{H}), 1.93(\mathrm{t}, J=7.4 \mathrm{~Hz}, 2 \mathrm{H}), 1.62-1.43(\mathrm{~m}, 4 \mathrm{H}), 1.34-1.20(\mathrm{~m}, 4 \mathrm{H}) .{ }^{13} \mathrm{C} \mathrm{NMR}(126$ $\left.\mathrm{MHz}, \mathrm{DMSO}-d_{6}\right) \delta 171.7(\mathrm{C}), 169.5(\mathrm{C}), 139.8(\mathrm{C}), 129.1(\mathrm{CH}), 123.4(\mathrm{CH}), 119.5(\mathrm{CH}), 36.8\left(\mathrm{CH}_{2}\right), 32.7\left(\mathrm{CH}_{2}\right)$, $28.9\left(\mathrm{CH}_{2}\right), 28.9\left(\mathrm{CH}_{2}\right), 25.5\left(\mathrm{CH}_{2}\right)$. Spectral data obtained was consistent with literature data. ${ }^{16}$

\section{Imidazole blocked O-isocyanate}

The in situ formation of imidazole blocked $O$-isocyanate was achieved following a literature procedure. ${ }^{17}$ O-Benzylhydroxylamine hydrochloride $(0.0970 \mathrm{~g}, 0.600 \mathrm{mmol})$ was suspended in THF $(0.6 \mathrm{M})$ and cooled to $0{ }^{\circ} \mathrm{C}$. The addition of carbonyldiimidazole $(0.0960 \mathrm{~g}, 0.600 \mathrm{mmol})$ was done over several minutes, upon which the solution was warmed to room temperature and stirred for 3 hours under inert atmosphere. The solid precipitate was then filtered from the solution. The filtrate was concentrated under reduced pressure and NMR analysis of the crude product supported the formation of the desired imidazole blocked derivative. The crude material was resuspended in $\mathrm{MeCN}(3.0 \mathrm{~mL}, 0.2 \mathrm{M})$. Benzoic acid $(0.0733 \mathrm{~g}, 0.600$ mmol) and stirred for 16 hours at room temperature or $120^{\circ} \mathrm{C}$. Reactions were concentrated under reduced pressure and analyzed using NMR. 


\section{NMR experiment}

A reaction following general procedure $A$ was conducted on a $0.2 \mathrm{mmol}$ scale in an NMR tube using DMSO$d_{6}$ as the reaction medium. 1,3,5-Trimethoxybenzene was used as an internal standard to monitor the reaction by ${ }^{1} \mathrm{H}$ NMR. Time point 0 corresponds to the initial reaction mixture taken at room temperature. The temperature in the NMR was then raised to $100{ }^{\circ} \mathrm{C}$. The first ${ }^{1} \mathrm{H}$ NMR at high temperature was obtained at 15 minutes, with subsequent spectra taken every 30 minutes for 20 hours.
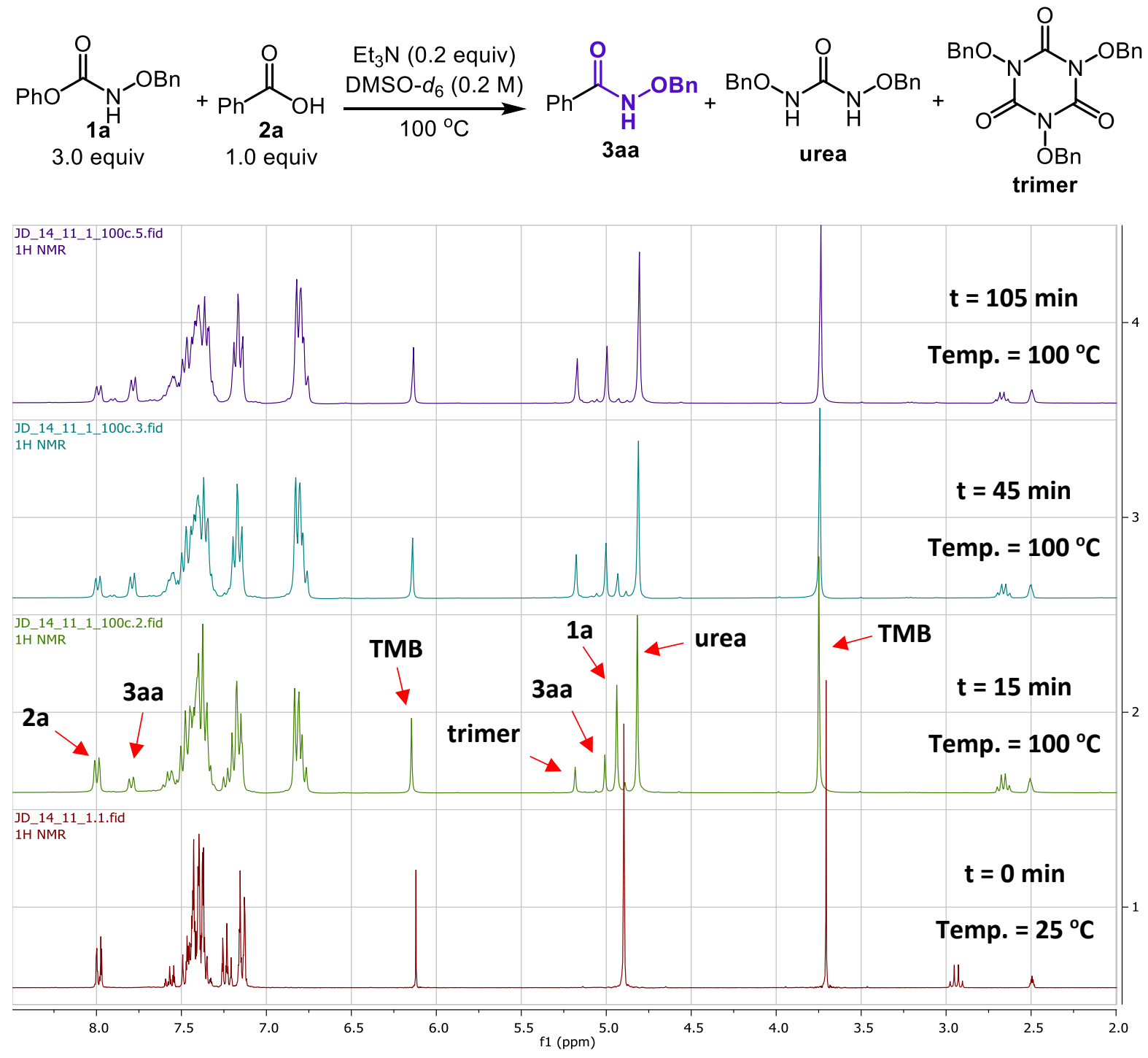

Figure S1. Representative ${ }^{1} \mathrm{H}$ NMR data obtained from monitoring study. 


\section{Selected Optimization Results}

Table S1: Solvent scan ${ }^{a}$

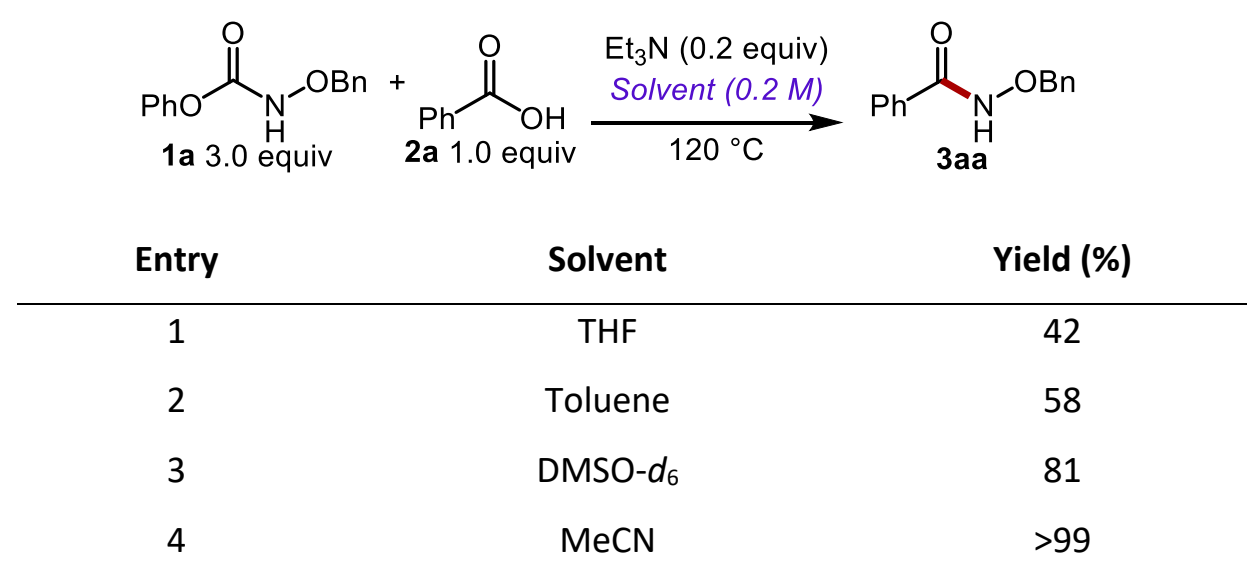

${ }^{a}$ Conditions: 1a $(0.6 \mathrm{mmol}), 2 \mathrm{a}(0.2 \mathrm{mmol}), \mathrm{Et}_{3} \mathrm{~N}(0.04 \mathrm{mmol})$, solvent $(0.2 \mathrm{M}), 120^{\circ} \mathrm{C} .{ }^{1} \mathrm{H}$ NMR yield determined by using 1,3,5-trimethoxybenzene as internal standard.

Table S2: Base scan ${ }^{\mathrm{a}}$

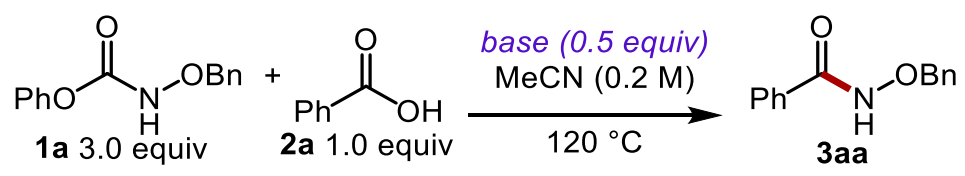

\begin{tabular}{ccc} 
Entry & Base & Yield (\%) \\
\hline 1 & $\mathrm{Et}_{3} \mathrm{~N}$ & $>99$ \\
2 & DIPEA & $>99$ \\
3 & $\mathrm{TMEDA}$ & 98 \\
4 & $N$-methylmorpholine & 93 \\
5 & $\mathrm{DBU}$ & 48 \\
6 & $\mathrm{~K}_{2} \mathrm{CO}_{3}$ & 88 \\
7 & $\mathrm{No} \mathrm{base}$ & $9 \%$
\end{tabular}

${ }^{a}$ Conditions: 1a $(0.6 \mathrm{mmol}), 2 \mathrm{a}(0.2 \mathrm{mmol})$, base $(0.1 \mathrm{mmol}), \mathrm{MeCN}(0.2 \mathrm{M}), 120{ }^{\circ} \mathrm{C} .{ }^{1} \mathrm{H}$ NMR yield determined by using $1,3,5-$ trimethoxybenzene as internal standard. 
Table S3: Blocking group scan ${ }^{a}$

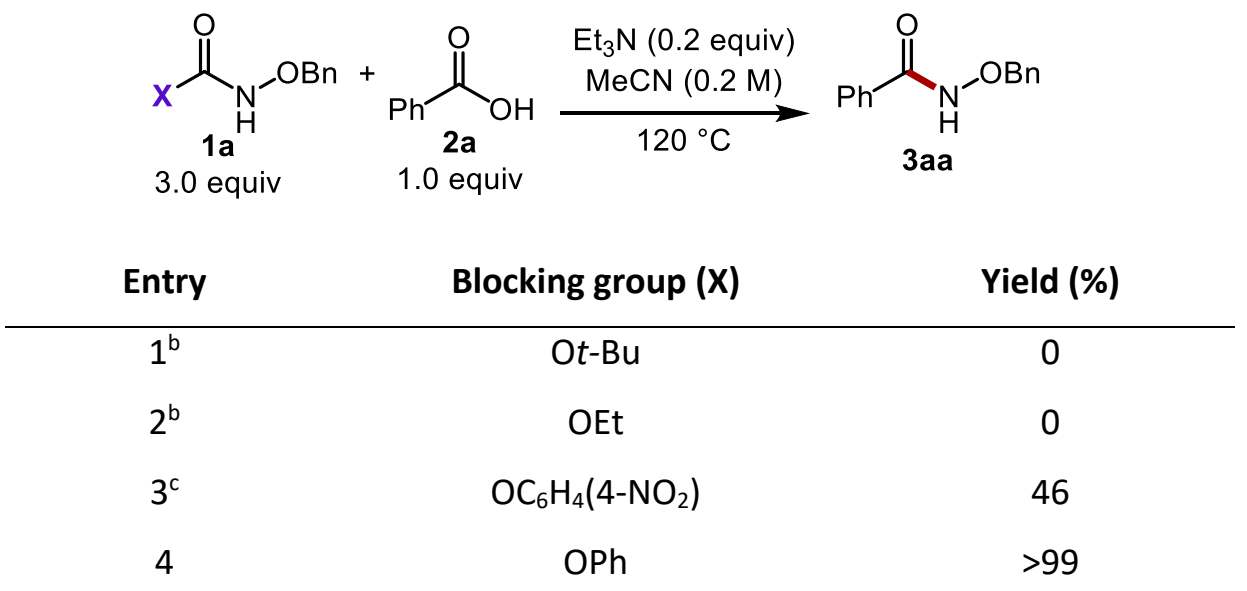

${ }^{a}$ Conditions: $1 \mathrm{a}(0.6 \mathrm{mmol}), 2 \mathrm{a}(0.2 \mathrm{mmol}), \mathrm{Et}_{3} \mathrm{~N}(0.04 \mathrm{mmol}), \mathrm{MeCN}(0.2 \mathrm{M}), 120{ }^{\circ} \mathrm{C} .{ }^{1} \mathrm{H}$ NMR yield determined by using $1,3,5-$ trimethoxybenzene as internal standard. ${ }^{b} \mathrm{O}$-Ethyl hydroxylamine derived starting material. ${ }^{\mathrm{C}}$ Reaction run at $80^{\circ} \mathrm{C}$ with $\mathrm{O}-\mathrm{methyl}$ hydroxylamine derived starting material. 

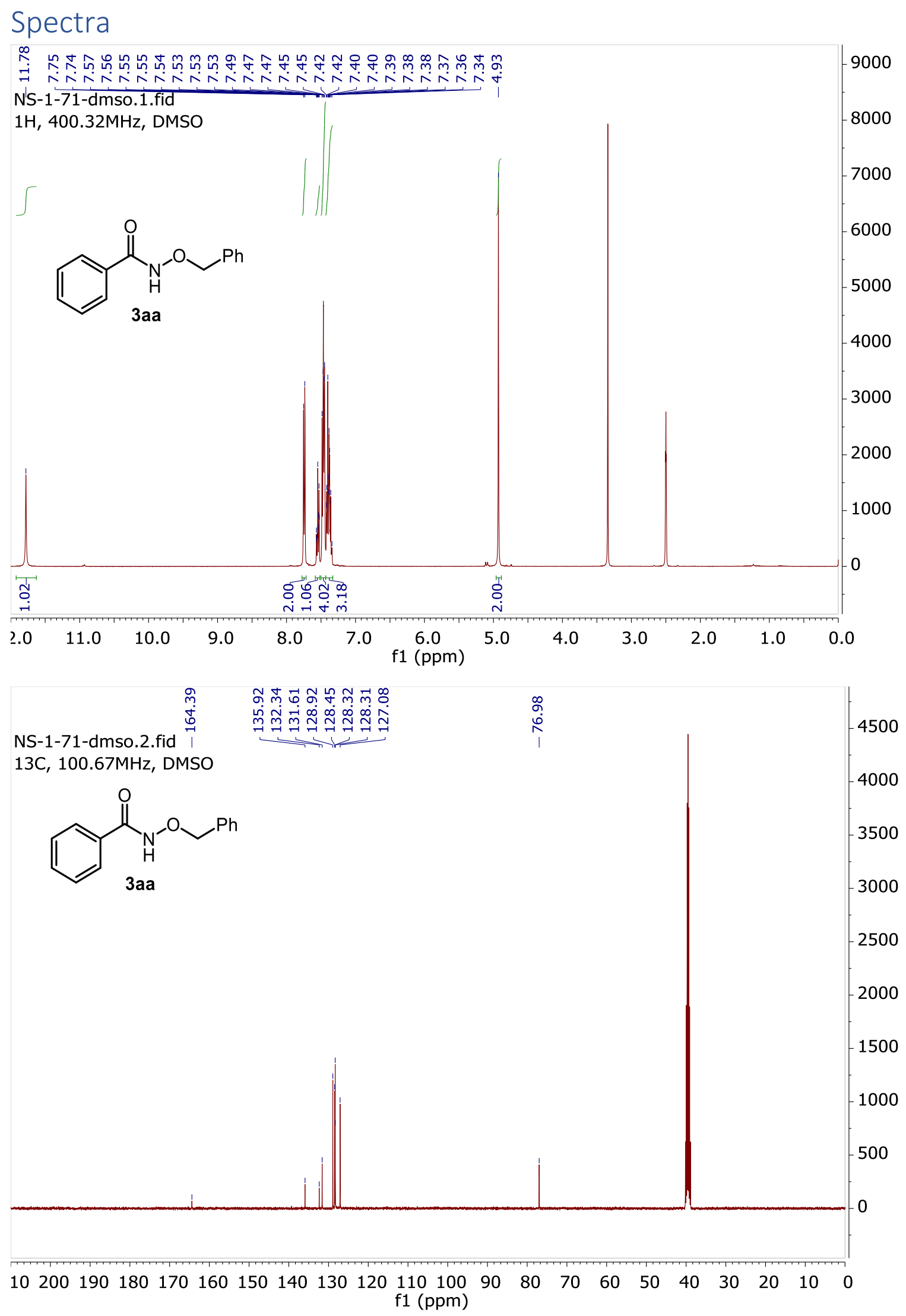


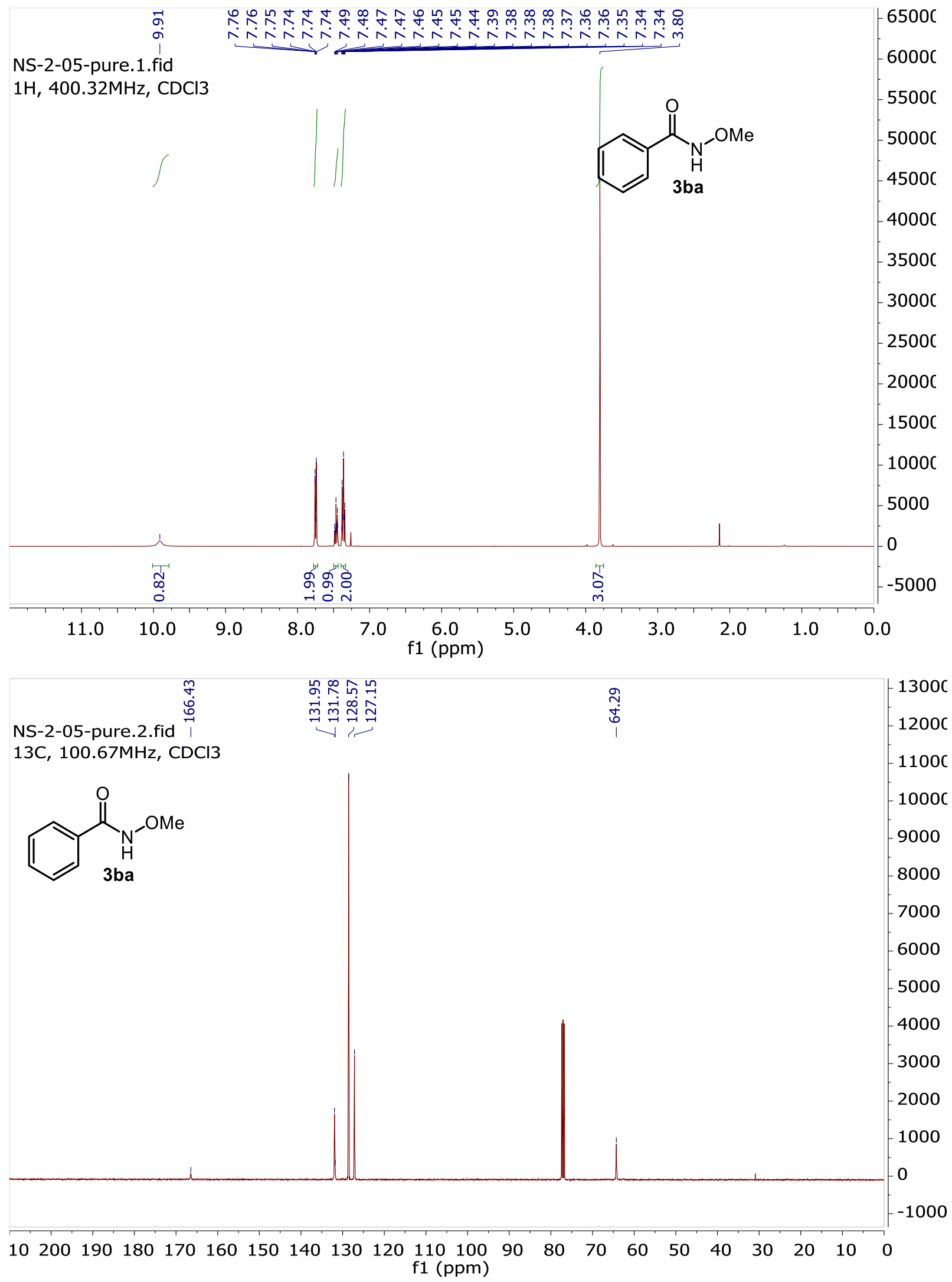




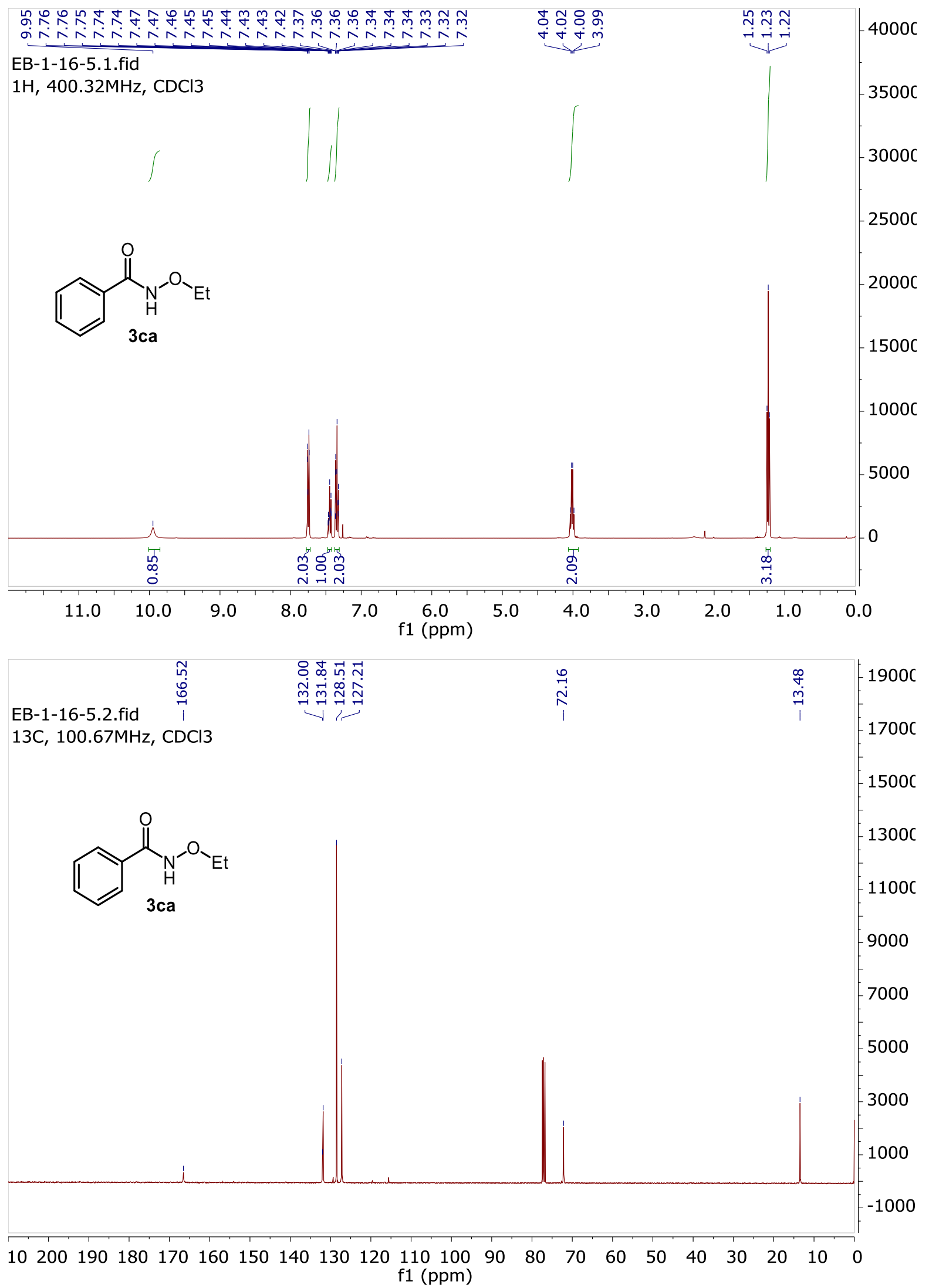



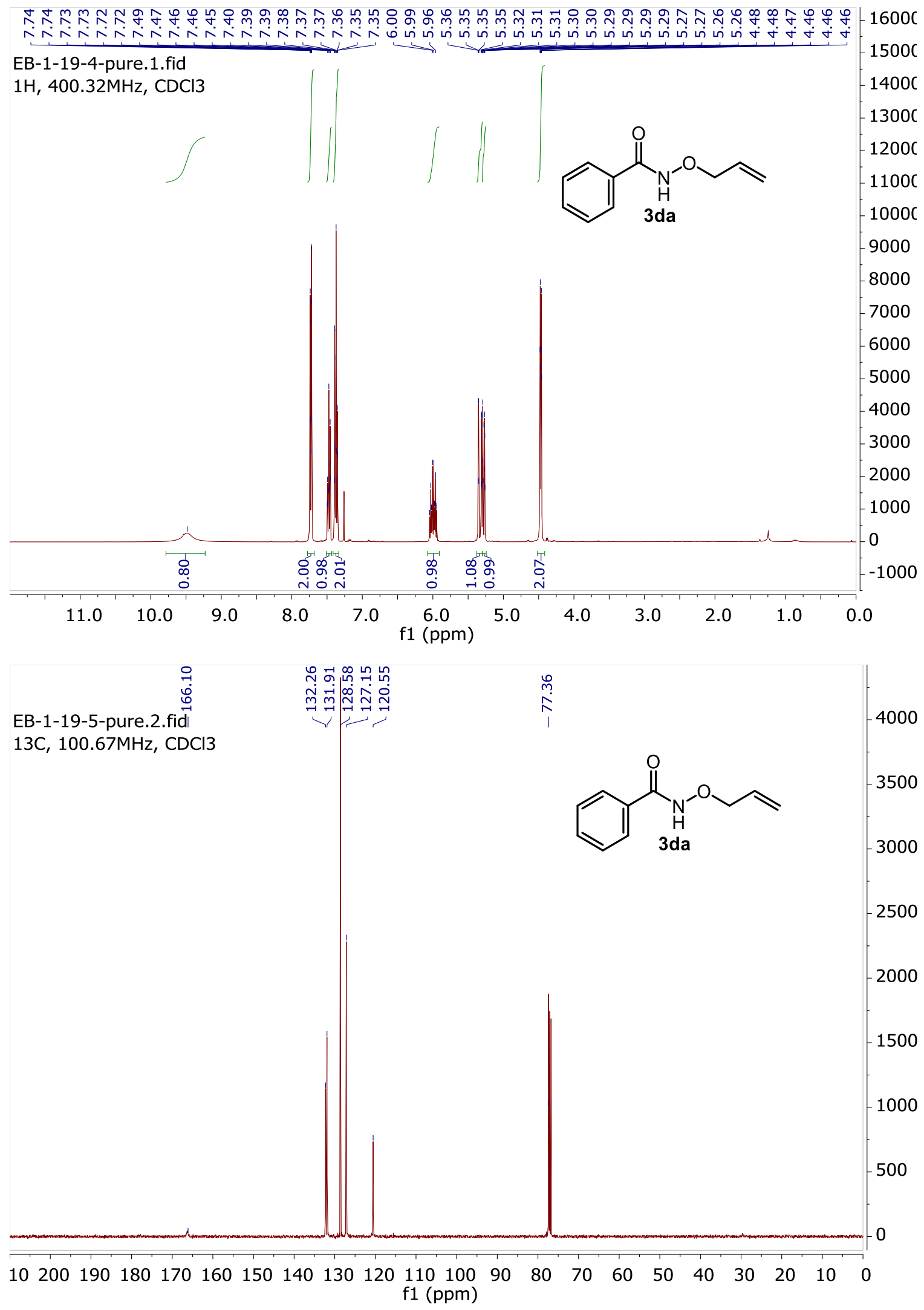


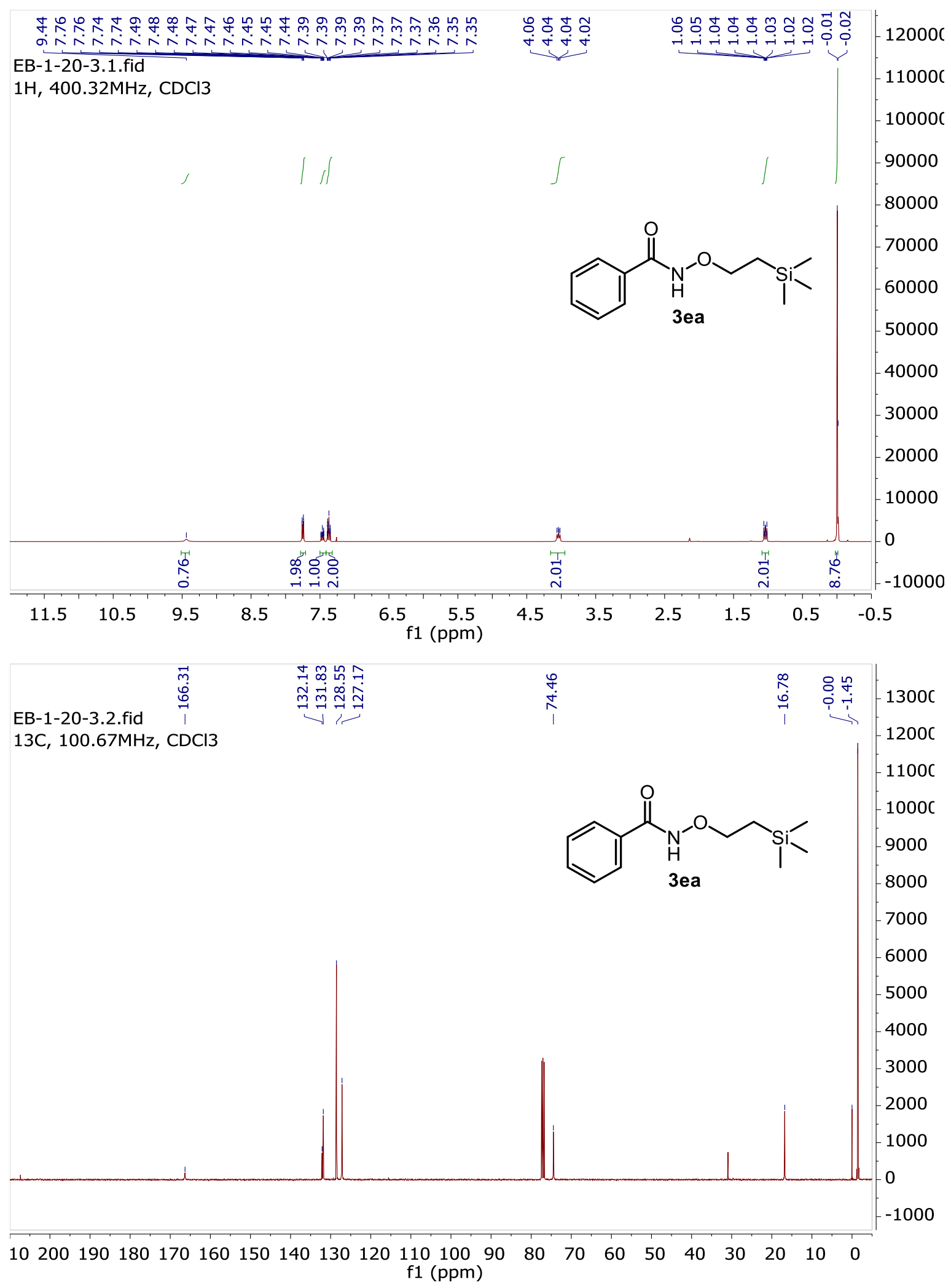




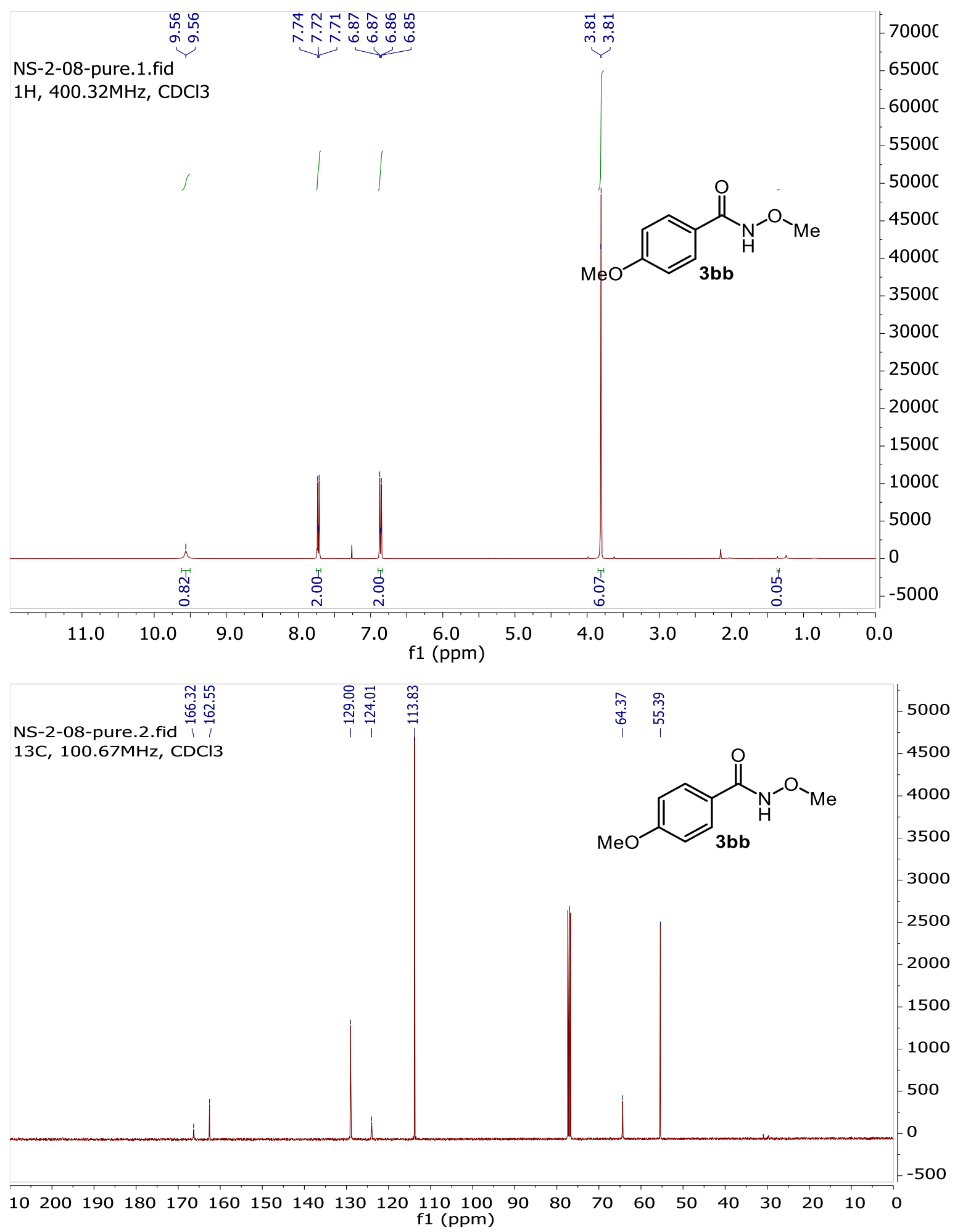




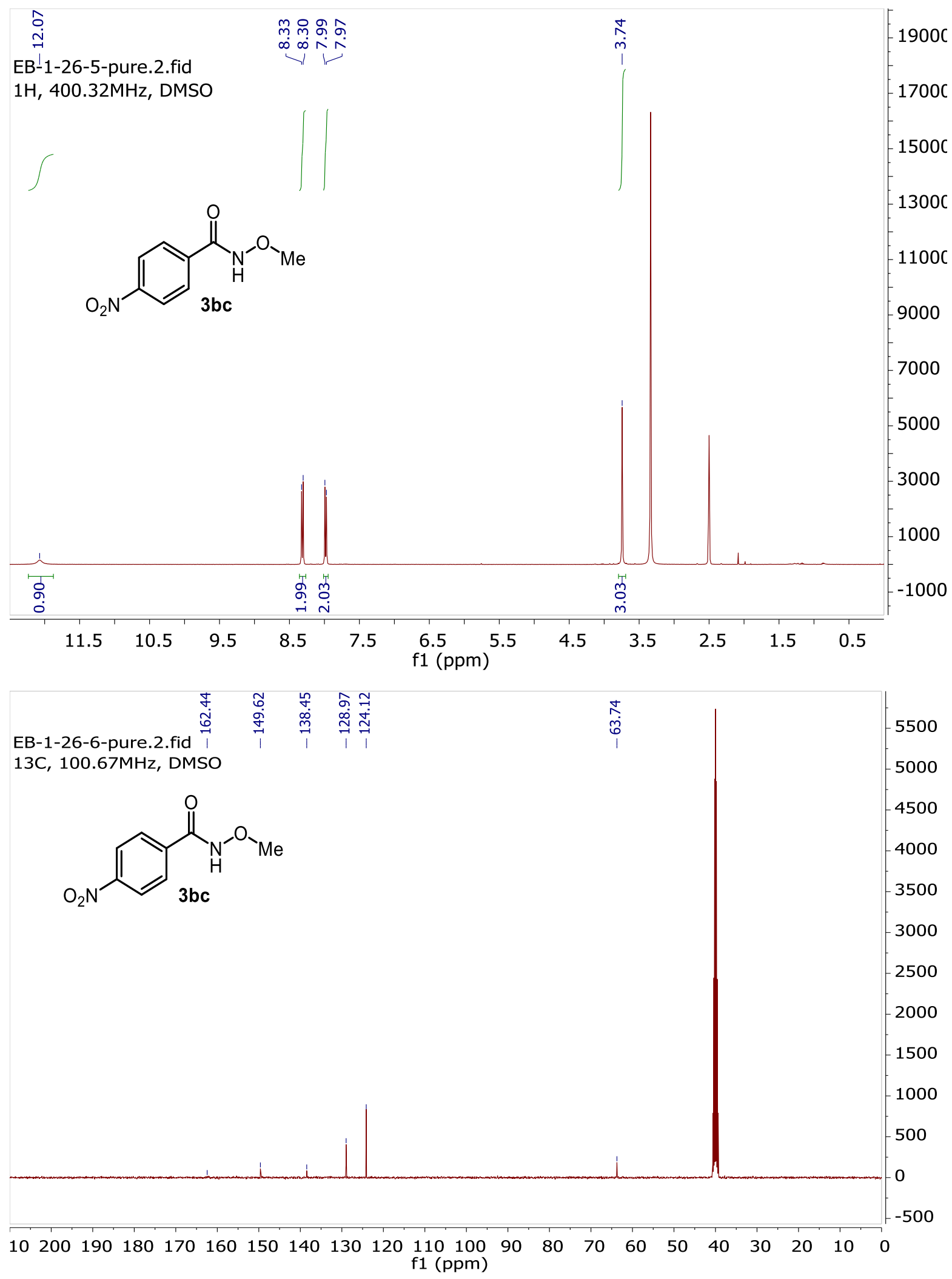




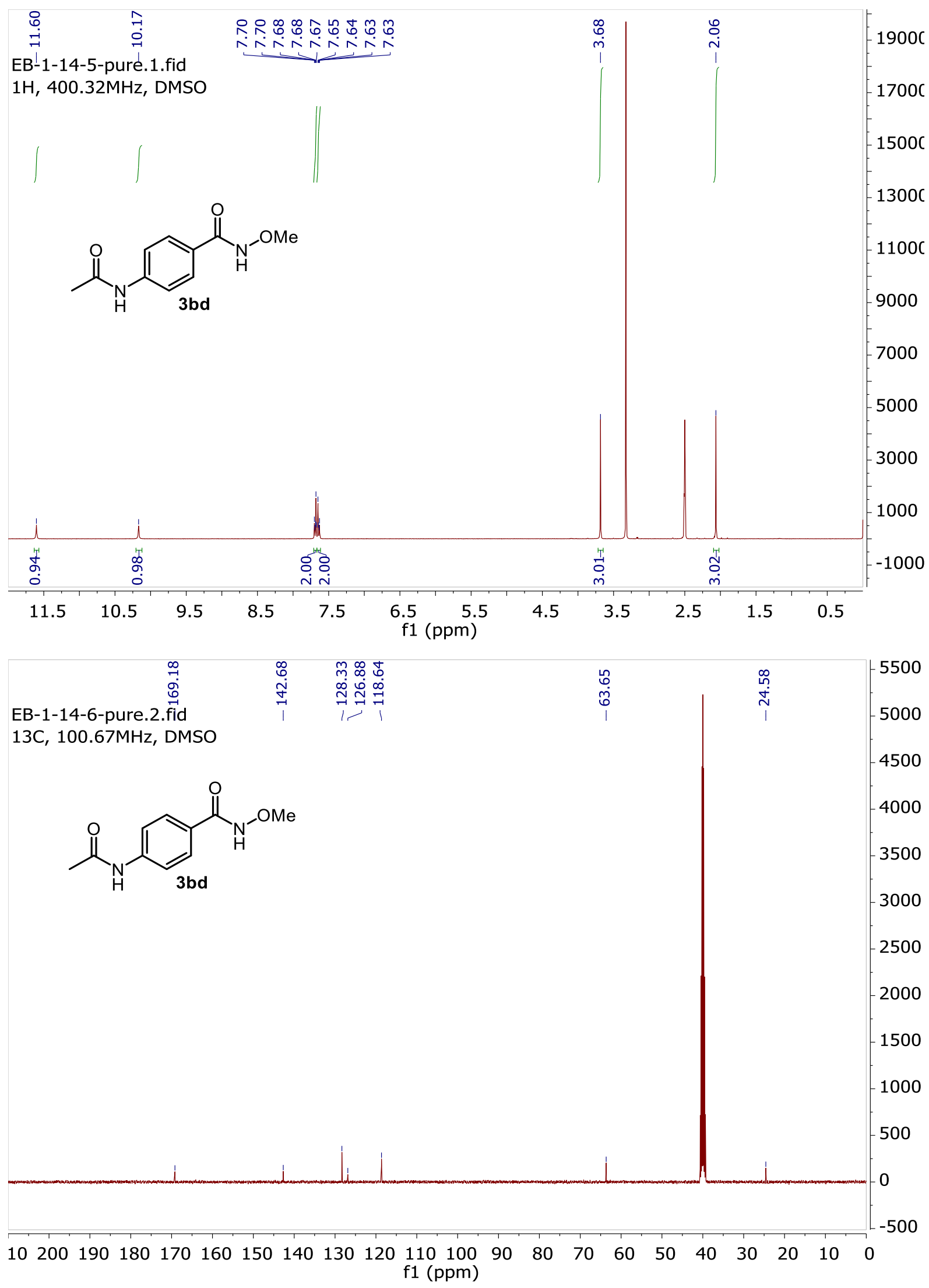



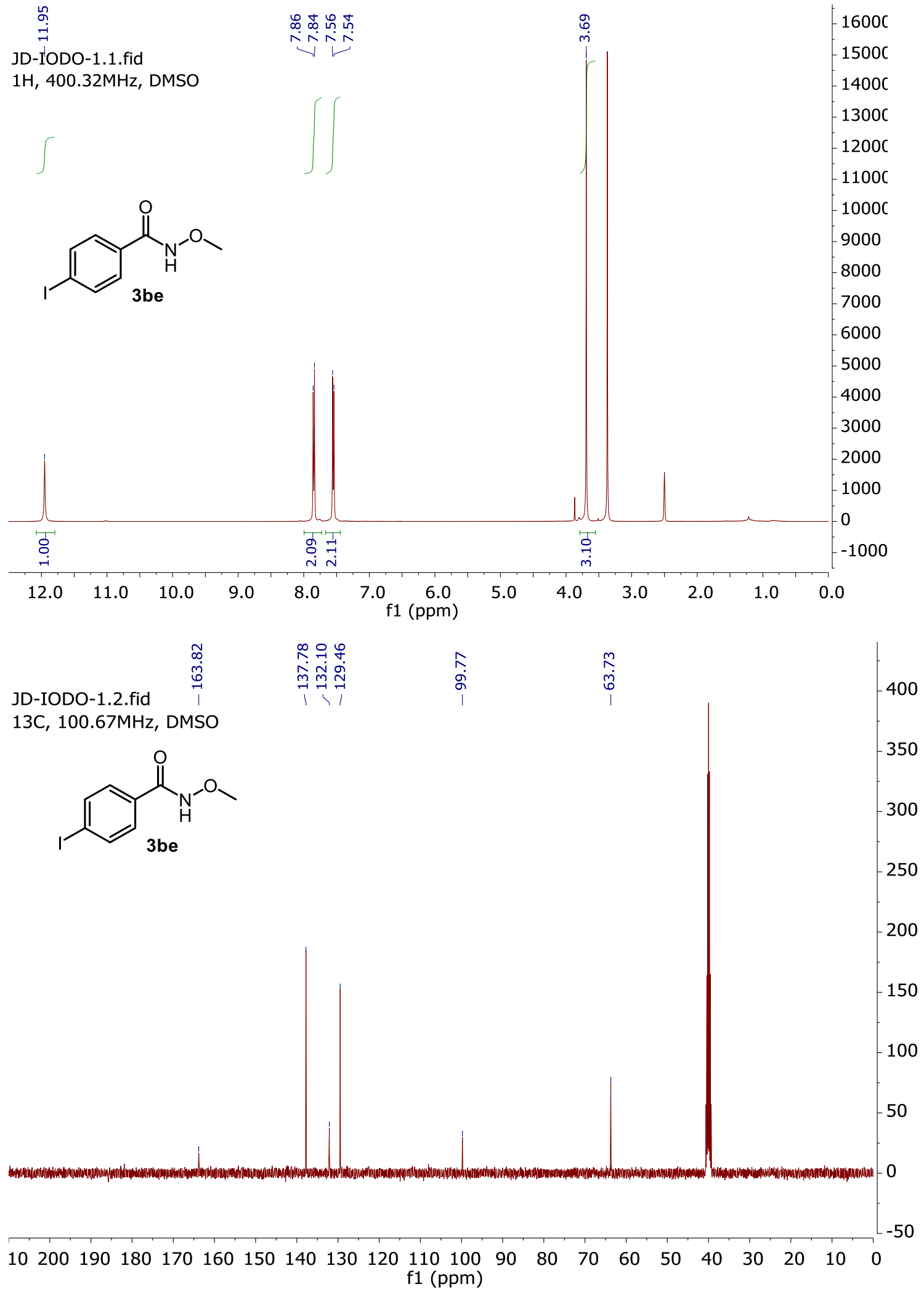


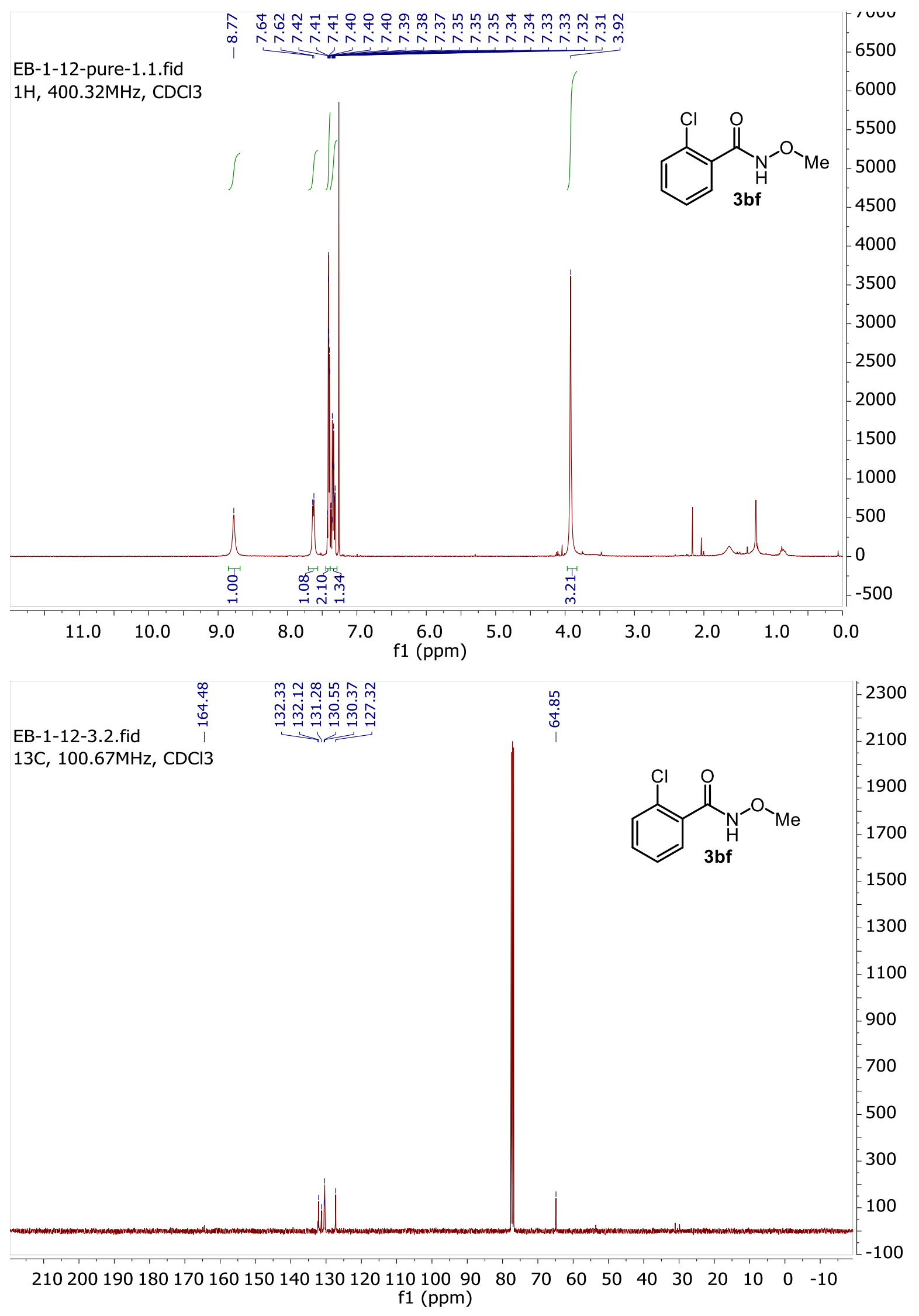



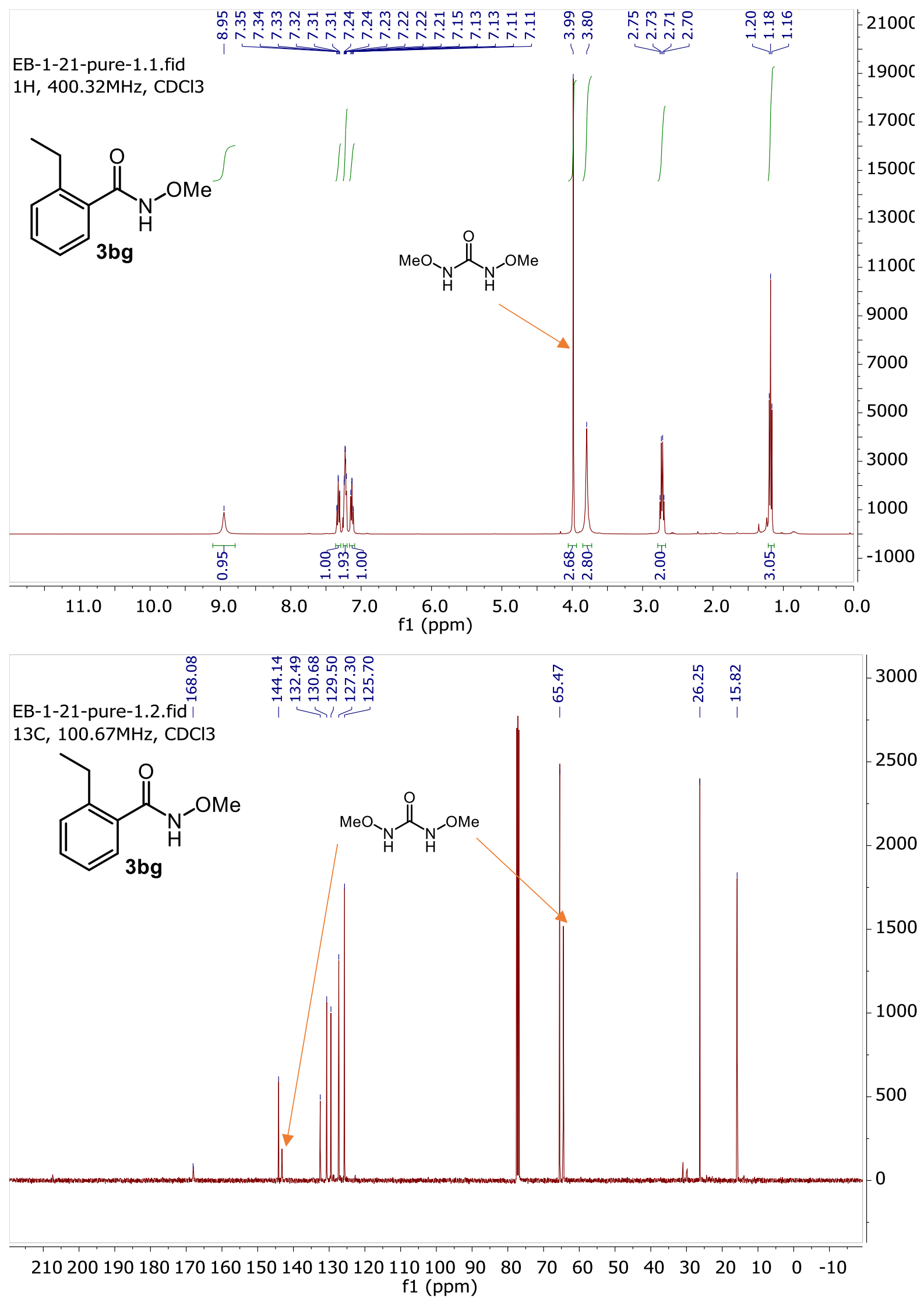


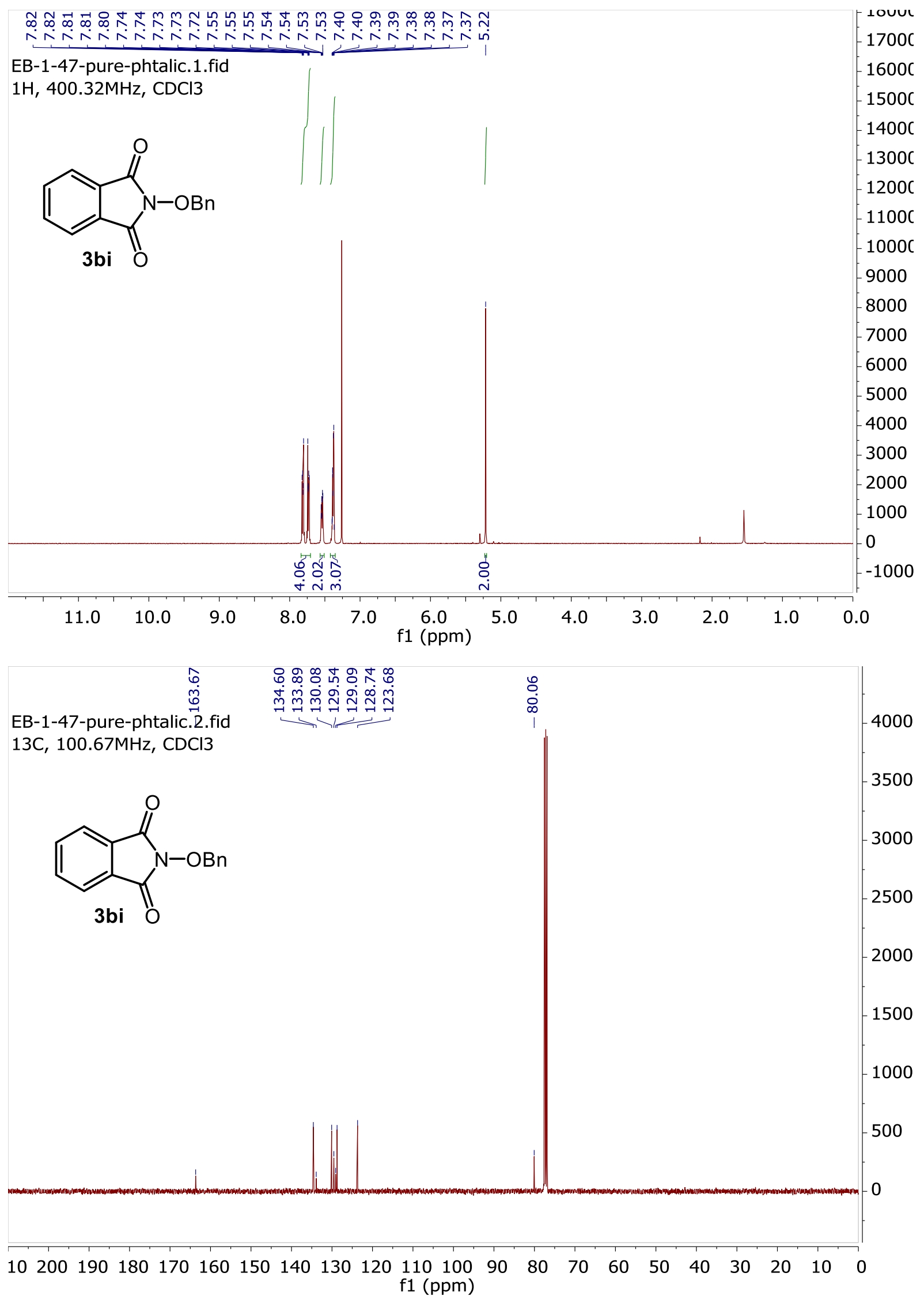




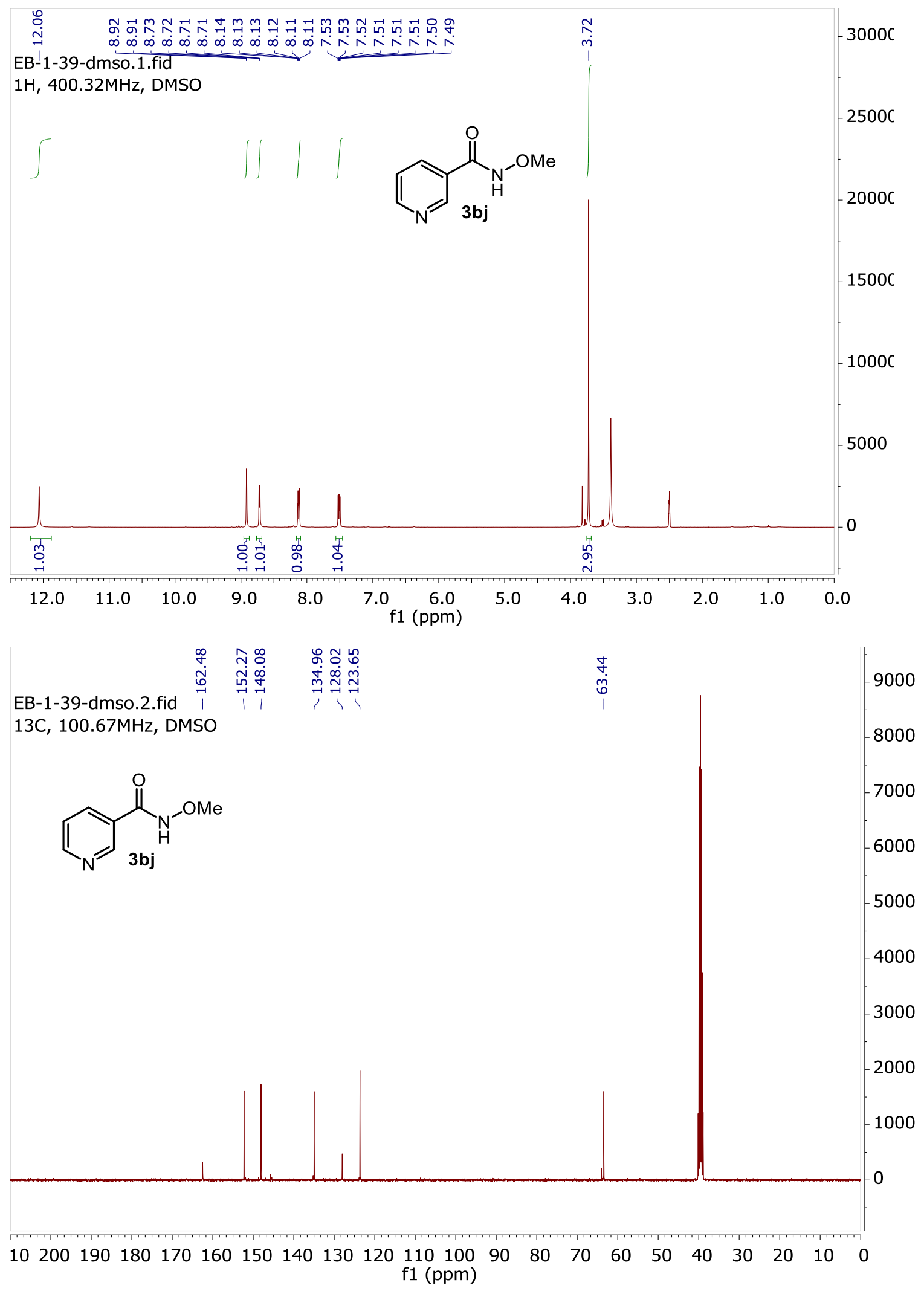



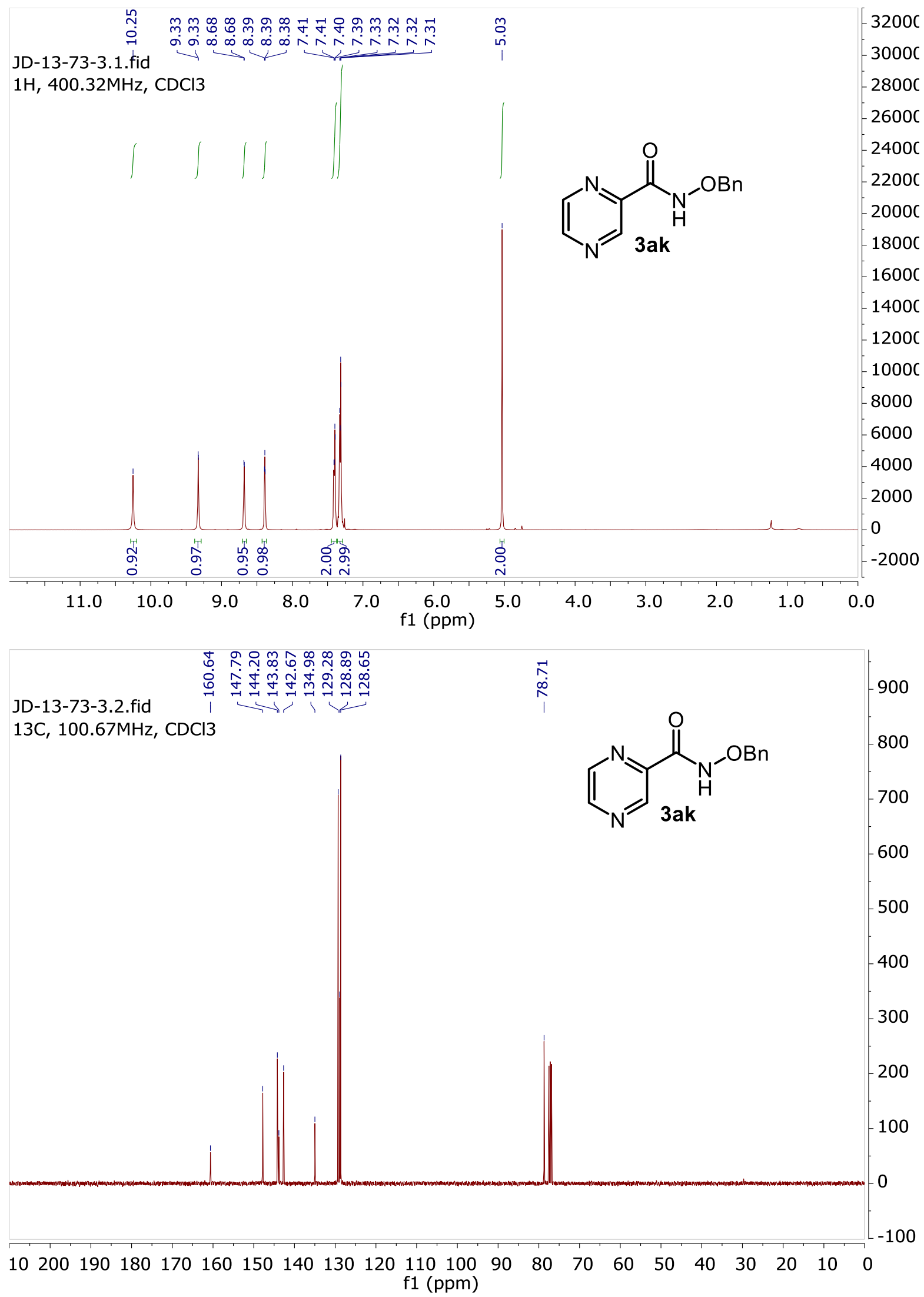


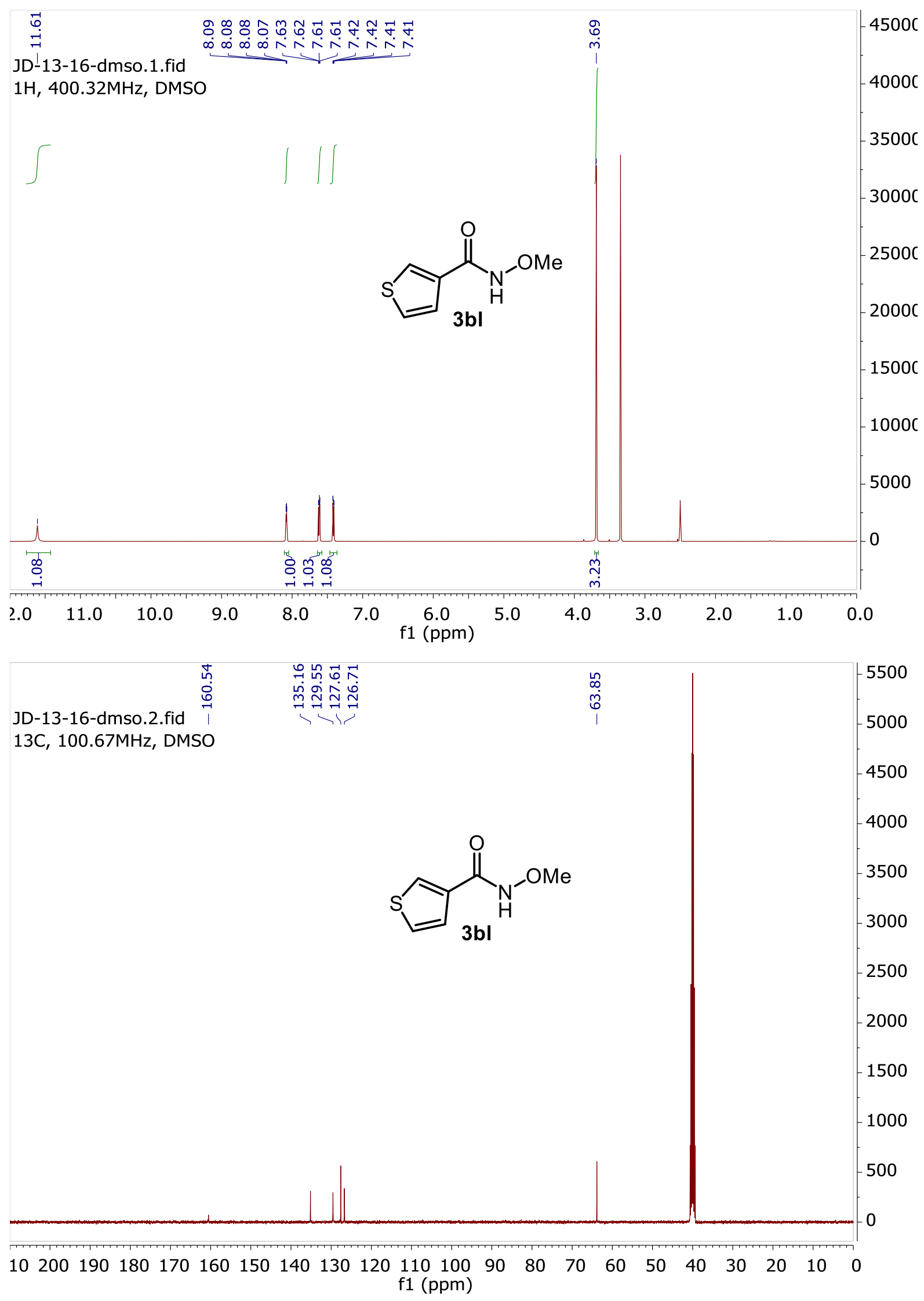




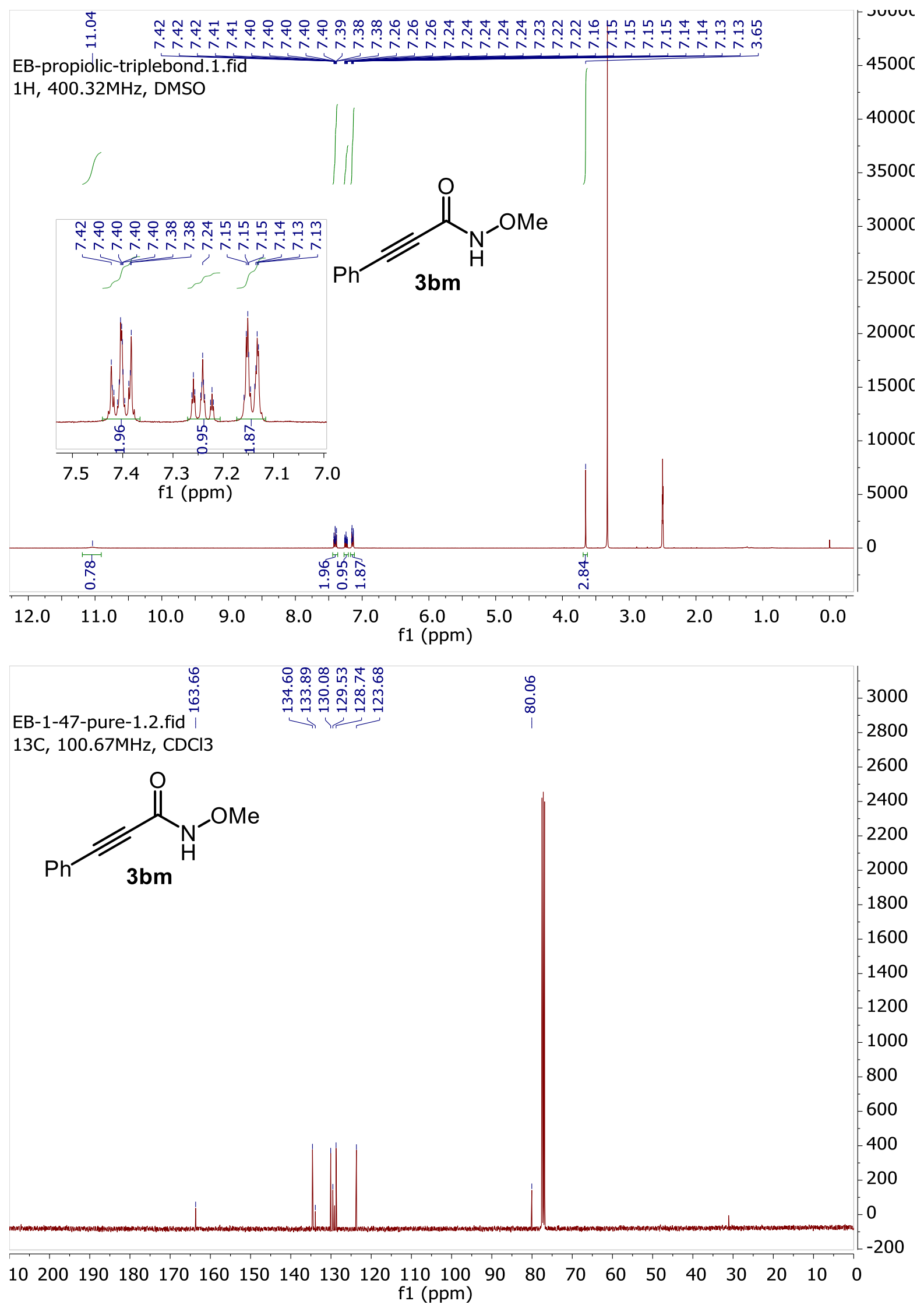




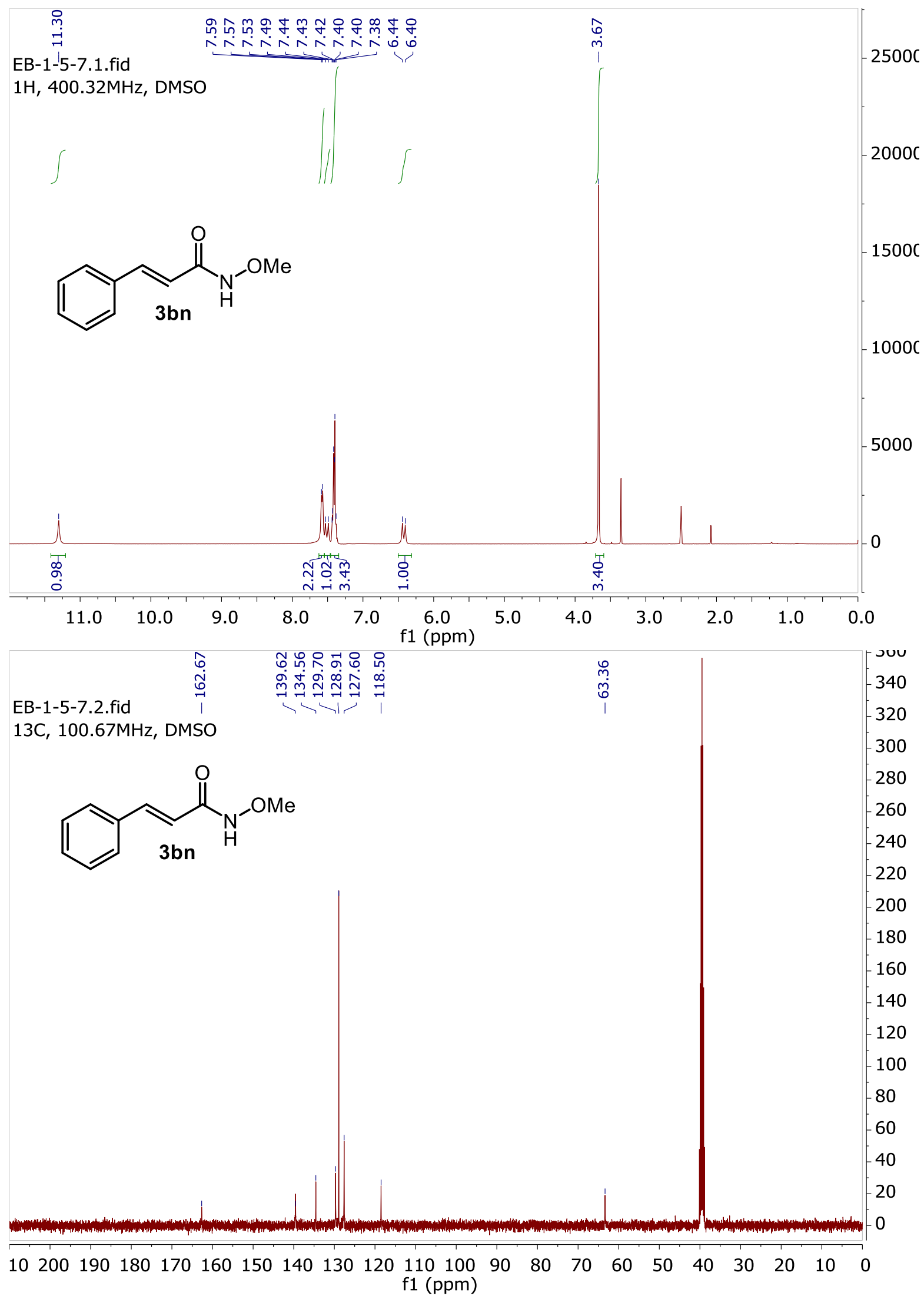




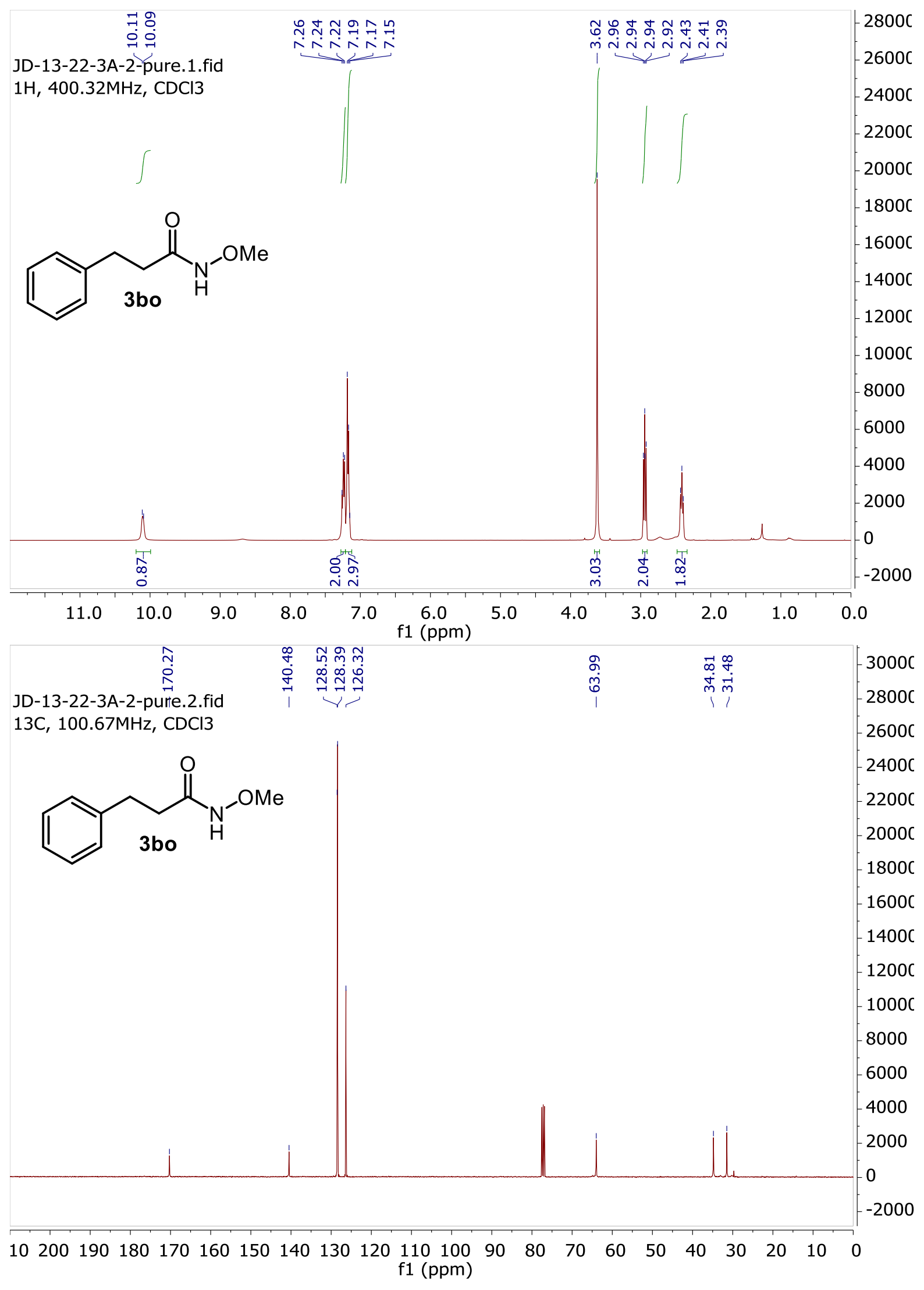




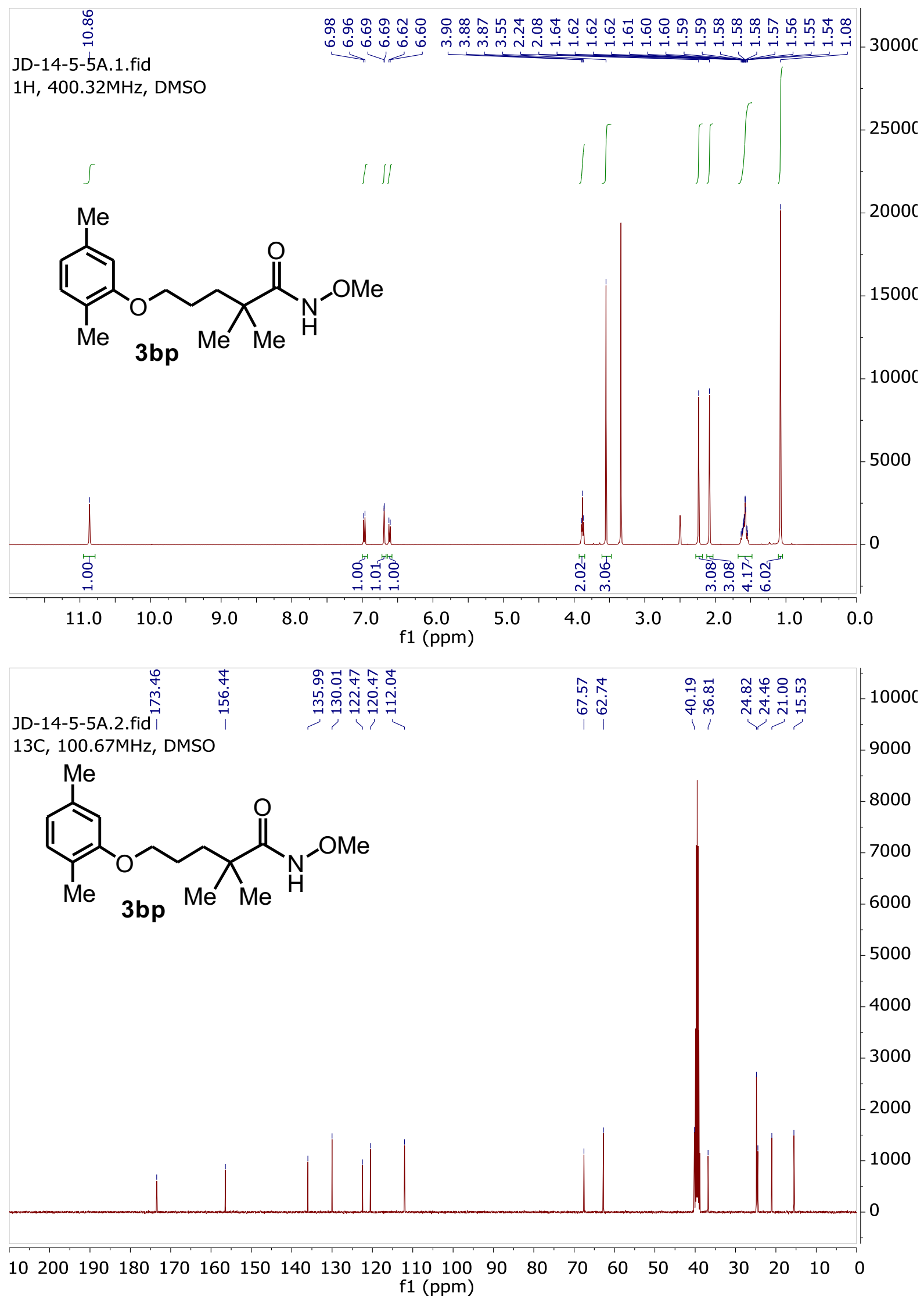




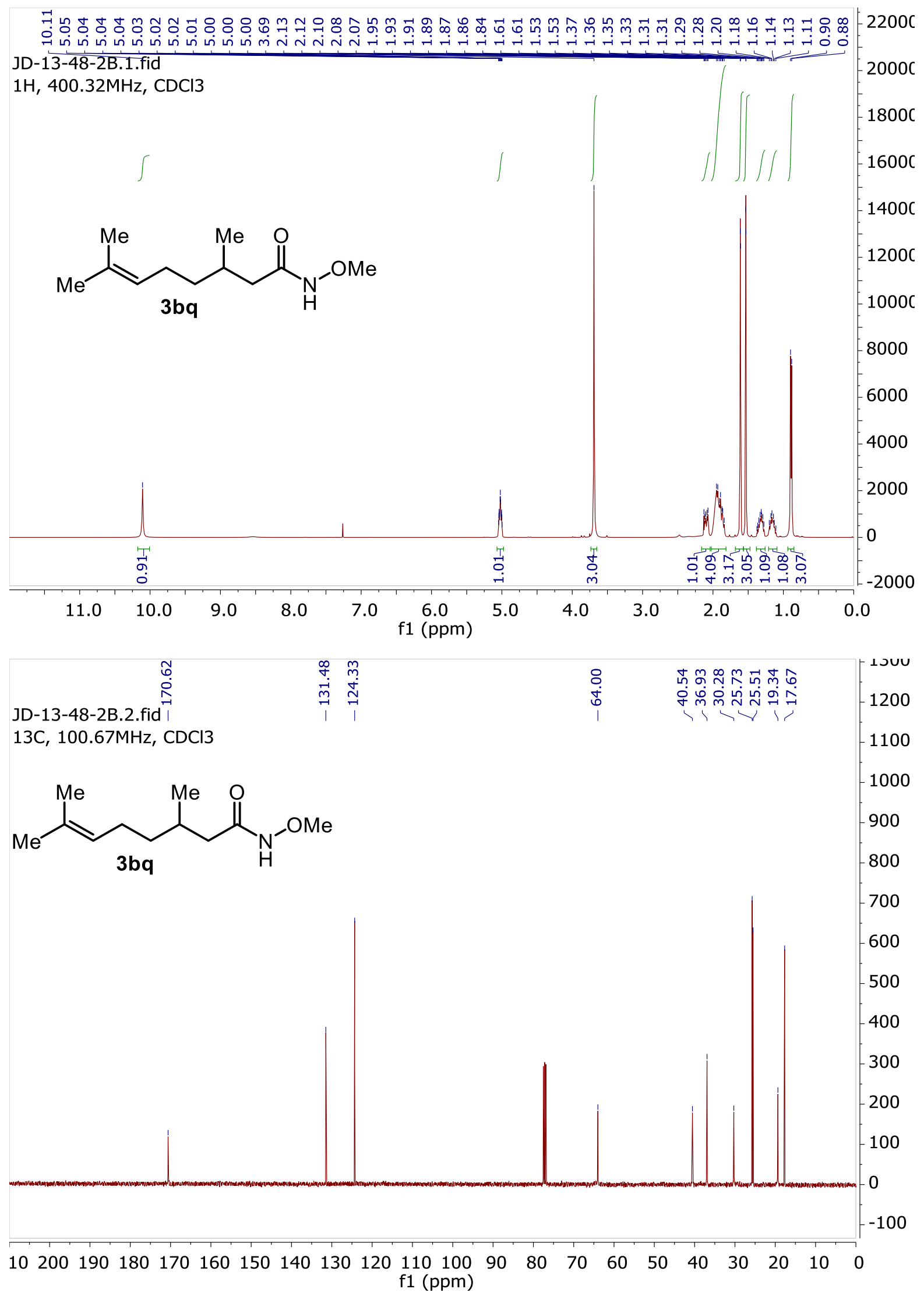




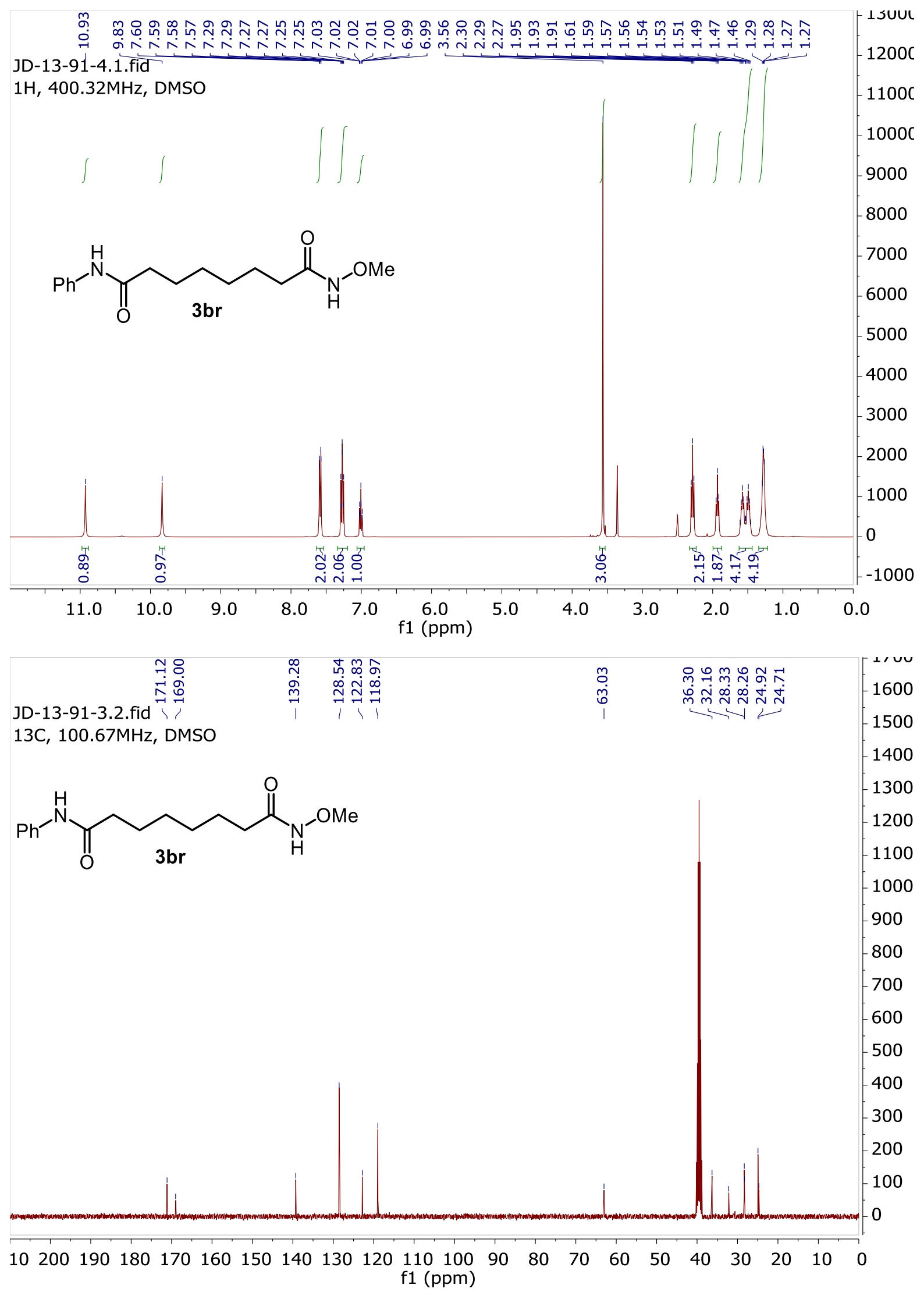




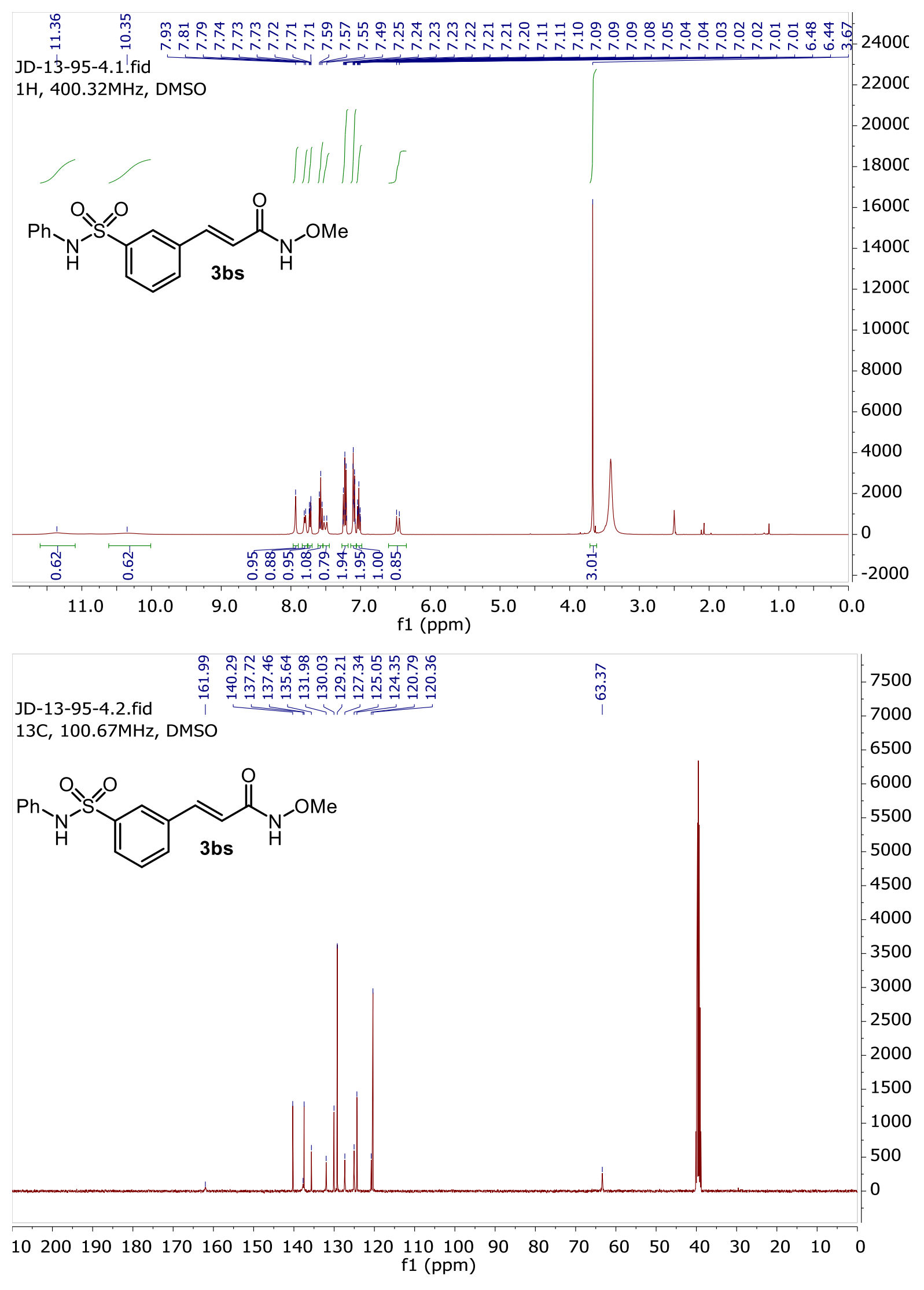




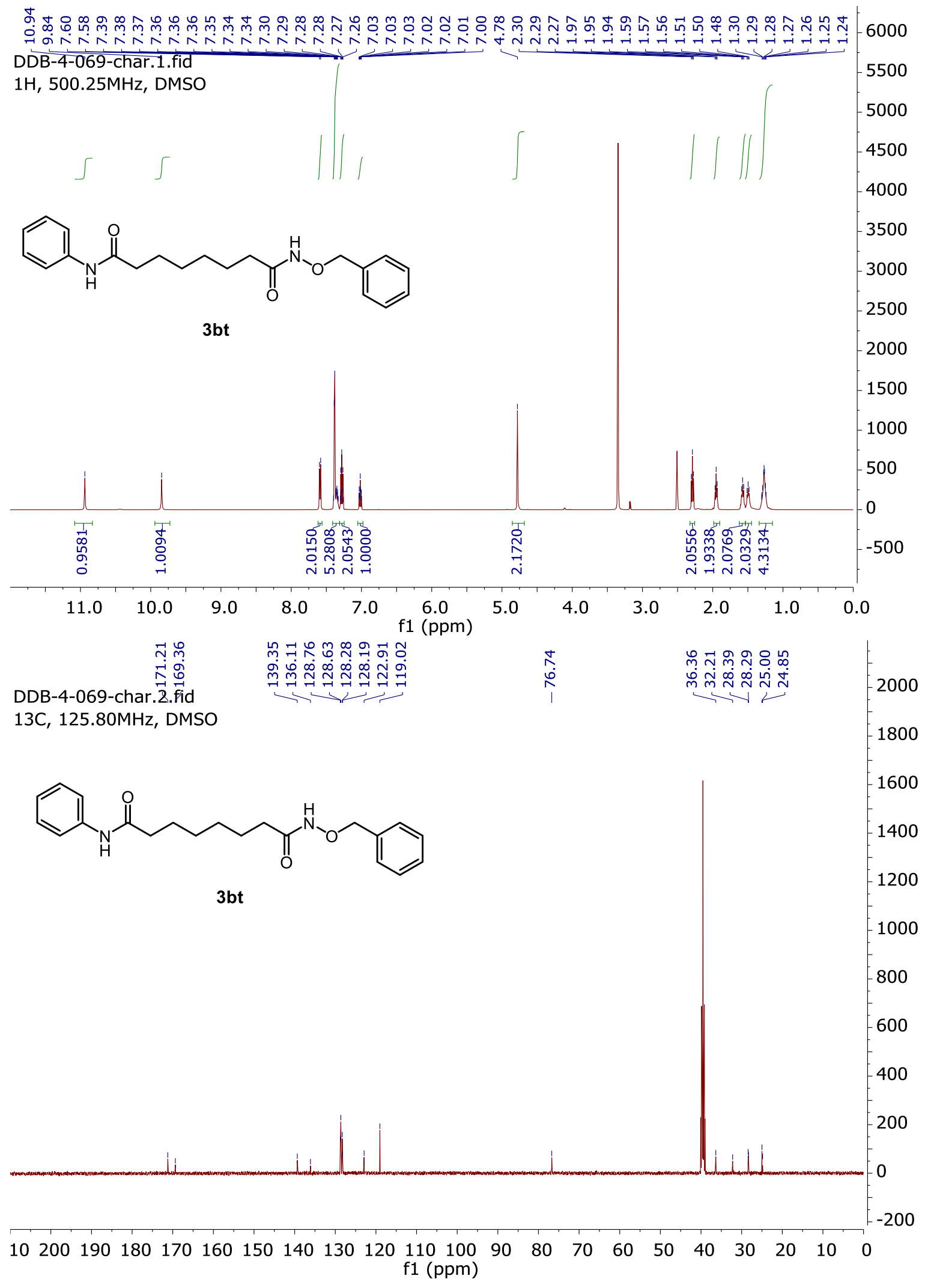



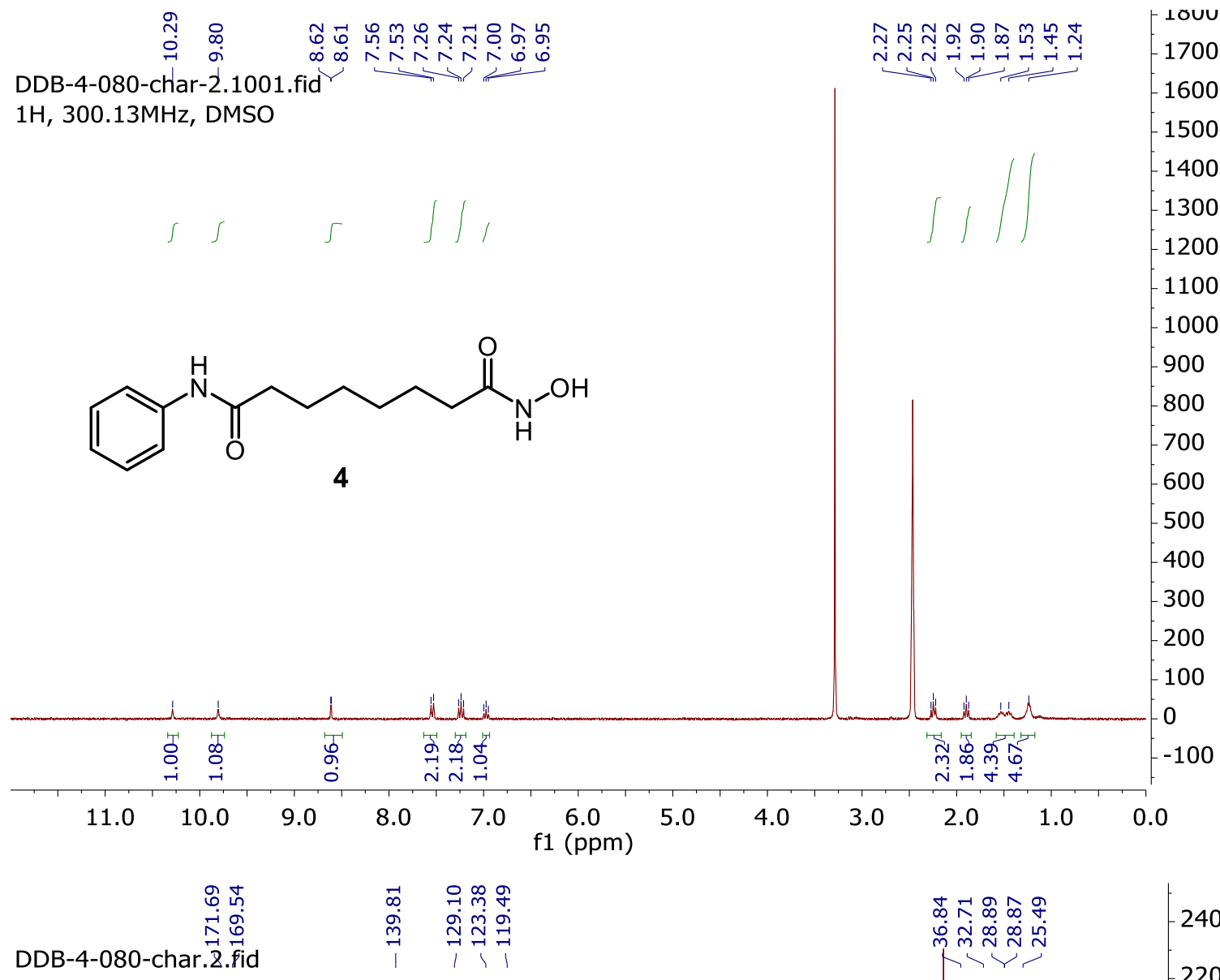

13C, $125.80 \mathrm{MHz}$, DMSO<smiles>O=C(CCCCCCC(=O)Nc1ccccc1)NO</smiles>

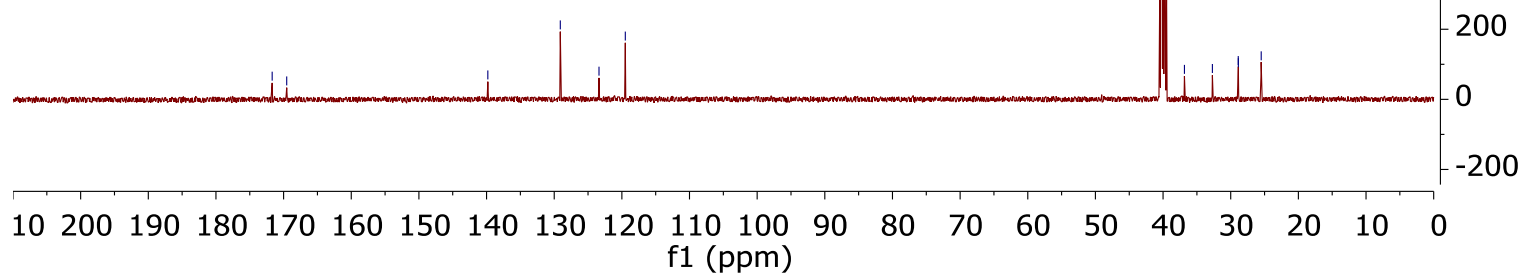




\section{References}

${ }^{1}$ Wang, Q.; An, J.; Alper, H.; Xiao, X-J.; Beauchemin, A. M. Chem. Commun. 2017, 53, 13055.

${ }^{2}$ Allen, M. A.; Ivanovich, R. A.; Polat, D. E.; Beauchemin, A. M. Org. Lett. 2017, 19, 6574.

${ }^{3}$ Mai, A.; Esposito, M.; Sbardella, G.; Massa, S. Org. Prep. Proced. Int. 2001, 33, 391.

${ }^{4}$ Reisch, H. A.; Leeming, P.; Raje, P. S. U.S.P. 8,642,809, February 4, 2014.

${ }^{5}$ Anumandla, D.; Littlefield, R.; Jeffrey, C. S. Org. Lett. 2014, 16, 5112

${ }^{6}$ Naruhisa, H.; Hyogo, H.-S. E.P. 1,666,470, July 6, 2006.

${ }^{7}$ Xu, Z.; Hu, W.; Wang, Z.; Guo, S. Eur. J. Med. Chem. 2017, 141, 211.

${ }^{8}$ Wrigglesworth, J. W.; Cox, B.; Lloyd-Jones, G. C.; Booker-Milburn, K. I. Org. Lett. 2011, 13, 5326.

${ }^{9}$ Samanta, R.; Bauer, J. O.; Strohmann, C.; Antonchick, A. P. Org. Lett. 2012, 14, 5518.

${ }^{10}$ Kukosha, T.; Trufilkina, N.; Belyakov, S.; Katkevics, M.; Synthesis 2012, 44, 2413.

${ }^{11}$ Zhao, J.; Li, H.; Li, P.; Wang, L. J. Org. Chem. 2019, 84, 9007.

${ }^{12}$ Yield calculated by ${ }^{1} \mathrm{H}$ NMR taking into account the urea by-product

${ }^{13}$ Zhu, C.; Liang, Y.; Hong, X.; Sun, H.; Sun, W.-Y.; Houk, K. N.; Shi, Z. J. Am. Chem. Soc. 2015, 137, 7564.

${ }^{14}$ Liang, D.; Yu, W.; Nguyen, N.; Deschamps, J. R.; Imler, G. H.; Li, Y.; Mackerell A. D., Jr.; Jiang, C.; Xue, F. J. Org. Chem. 2017, 82, 3589.

${ }^{15}$ Rubio-Ruiz, Belen. Bioorthogonal Compounds Comprising a Propargyl Group for Treating Cancer. WO 2017/199028 A1, 2017.

${ }^{16}$ Papadopoulos, G. N.; Kokotos, C. G. Chem. Eur. J. 2016, 22, 6964.

${ }^{17}$ Daicel chemical industries, E.P. 1,666,470, June $7^{\text {th }}, 2006$. 